\title{
THE PRINCIPAL 2-BLOCKS OF FINITE GROUPS WITH ABELIAN SYLOW 2-SUBGROUPS
}

\author{
By \\ Shigeo KosHITANI
}

\section{Introduction}

Let $G$ be a finite group, $p$ a prime number and $B$ a $p$-block of $G$ with defect group $D$. There is an important problem in representation theory of finite groups that is to give a description of $B$ when the structure of $D$ is given. Concerning with this problem there are some successful results. E. C. Dade [9] proved his results when $D$ is cyclic. R. Brauer [6] proved his results for the case where $p=2$ and $D$ is dihedral by making use of his powerful methods ([3], [4], [5]). Using Brauer's methods J. B. Olsson [18] obtained his results when $p=2$ and $D$ is generalized quaternion or quasidihedral. In $[3$, IV] R. Brauer investigated $B$ when $p=2$ and $D$ is elementary abelian of order 4 .

In the present paper we study $B$ when $p=2$ and $B$ is the principal 2-block of $G$ with an abelian Sylow 2-subgroup $P$. Let $e(G)=\left|N_{G}(P): C_{G}(P)\right|$. Let $B_{0}(G)$ be the principal 2-block of $G$, and let $O(G)$ and $O^{\prime}(G)$ be the maximal normal subgroup of $G$ of odd order and the minimal normal subgroup of $G$ of odd index, respectively. By the results on finite groups with abelian Sylow 2-subgroups ([2], [16], [17], [20], [21]), the structure of $O^{\prime}(G / O(G))$ is almost determined. In general, however, $B_{0}(G)$ is different from $B_{0}(S)$ where $S=$ $O^{\prime}(G / O(G))$. The main purpose of this paper is to investigate the relation between $B_{0}(G)$ and $B_{0}(S)$. In particular we shall prove that $B_{0}(G)$ is isomorphic to $B_{0}(S)$ for the cases where $e(G)=e(S)=$ prime, 9 and 21 .

In section 1 we shall state several lemmas and propositions which will be useful for our aim. One of them is Alperin's theorem on isomorphic principal blocks [1]. Let $S=O^{\prime}(G / O(G))$. In section 2 we shall consider $B_{0}(G)$ for the case where $e(G)=2^{m}-1$. In particular, we shall prove that if $G$ is nonsolvable and if $e(G)$ is prime then $e(G)=2^{m}-1$ for some $m \geqq 2$ and $B_{0}(G)$ is isomorphic to $B_{0}(S)$. In sections 3 and 4 we shall investigate $B_{0}(G)$ for the cases when $e(G)=9$ and 21 , respectively. Indeed, we shall prove that if $e(G)=e(S)=9$ or 21 then $B_{0}(G)$ is isomorphic to $B_{0}(S)$. It is noted that when $e(G) \neq e(S), B_{0}(G)$ is not necessarily isomorphic to $B_{0}(S)$. In sections 5 and 6 we shall determine Received October 17, 1979 
$B_{0}(G)$ when $P$ is elementary abelian of order 8 and 16 , respectively.

Throughout this paper we shall use the following notation. When $S$ is a subset of $G, N_{G}(S)$ and $C_{G}(S)$ denote the normalizer and the centralizer of $S$ in $G$, respectively. Specially, for each $x \in G$ we write $C_{G}(x)$ for $C_{G}(\{x\})$. If $x, y \in G$, we write $x^{y}$ for $y^{-1} x y$. When $S$ is a subset of $G,\langle S\rangle$ denotes the subgroup of $G$ generated by $S$. When $x_{1}, \cdots, x_{n}$ are elements of $G$ and $S$ is a subset of $G$, we also write $\left\langle x_{1}, \cdots, x_{n}, S\right\rangle$ for the subgroup of $G$ generated by $\left\{x_{1}, \cdots, x_{n}\right\} \cup S$. The cyclic group of order $n$ is denoted $Z_{n}$ for a positive integer $n$. We write $G^{\prime}$ and $Z(G)$ for the commutator subgroup of $G$ and the center of $G$, respectively. We denote by $\operatorname{Aut}(G)$ the group of all automorphisms of $G$. Let us denote by $O_{p^{\prime}}(G)$ the maximal normal subgroup of $G$ of order prime to $p$, and by $O^{p^{\prime}}(G)$ the minimal normal subgroup of $G$ of index prime to $p$. In particular, for $p=2$ we write $O(G)$ and $O^{\prime}(G)$ for $O_{2^{\prime}}(G)$ and $O^{2 \prime}(G)$, respectively. When $P$ is an abelian Sylow 2-subgroup of $G$, we write $e(G$ ) (or shortly $e$ ) for $\left|N_{G}(P): C_{G}(P)\right|$. When $B$ is a $p$-block of $G$, let us denote by $\operatorname{Irr}(B)$ the set of all irreducible complex characters in $B$, by $\operatorname{IBr}(B)$ the set of all irreducible Brauer characters in $B$, by $k(B)$ the number of elements of $\operatorname{Irr}(B)$, by $k^{\prime}(B)$ the number of elements of $\operatorname{Irr}(B)$ with degree one, and by $l(B)$ the number of elements of $\operatorname{IBr}(B)$. We write $B_{0}(G)$ (or shortly $B_{0}$ ) for the principal p-block of $G$, and for each $x \in G$ we write $b_{x}$ for $B_{0}\left(C_{G}(x)\right)$. When $\psi_{1}$ and $\phi_{2}$ are complex characters of $G$, let $\left(\phi_{1}, \phi_{2}\right)=(1 /|G|) \Sigma_{g \in G} \phi_{1}(g) \psi_{2}\left(g^{-1}\right)$, that is to say, $\left(\phi_{1}, \phi_{2}\right)$ is the inner product of $\phi_{1}$ and $\phi_{2}$. We write $1_{G}$ for the trivial complex (or Brauer) character of $G$. When $H$ is a normal subgroup of $G,\left.\phi\right|_{H}$ denotes the restriction of $\psi$ to $H$ for a character $\psi$ of $G,\left.W\right|_{H}$ denotes the restriction of $W$ to $H$ for a representation $W$ of $G$, and $I_{G}(\tilde{\psi})$ denotes the inertial group of $\tilde{\psi}$ in $G$ for a character $\tilde{\psi}$ of $H$, that is to say, $I_{G}(\tilde{\psi})=\left\{g \in G \mid \tilde{\phi}^{g}=\tilde{\phi}\right\}$, where $\tilde{\phi}^{g}$ is the conjugate of $\tilde{\psi}$.

\section{Preliminaries}

In this section we state some lemmas and propositions which will be needed for our aim. We fix a prime number $p$ and we consider $p$-modular representations of a finite group $G$.

LEMMA 1.1. Let $G$ be a finite group with a Sylow p-subgroup $P$, and let $K=O_{p^{\prime}}(G), \bar{G}=G / K$ and $\bar{P}=(P K) / K$. Then we have the following.

(i) $B_{0}(G)=B_{0}(\bar{G})$.

(ii) $N_{G}(P) / C_{G}(P) \cong N_{\bar{G}}(\bar{P}) / C_{\bar{G}}(\bar{P})$. 
Proof. We get (i) by [10, Theorem 65.2] and [11, V (4.3)]. Since $N_{\bar{G}}(\bar{P})=$ $\left(N_{G}(P) \cdot K\right) / K$ from $[15$, I 7.7 Hilfssatz $(\mathrm{c})]$ and since $C_{\bar{G}}(\bar{P})=\left(C_{G}(P) \cdot K\right) / K$ from $[19$, Lemma 2.2$]$, we easily get (ii).

We shall frequently use the next four propositions in order to prove our main theorems.

Proposition 1.2. (Brauer). Let $G=Q C_{G}(Q)$ where $Q$ is a p-group, and let $\bar{G}=G / Q$. Then $l\left(B_{0}(G)\right)=l\left(B_{0}(\bar{G})\right)$.

Proof. See [10, Lemma 64.5 and Theorem 65.2(2)].

Proposition 1.3 (Brauer). Let $H$ be a normal subgroup of $G$. If $W$ is an ordinary or modular irreducible representation in $B_{0}(G)$, then any irreducible constituent of $\left.W\right|_{H_{\text {. }}}$ lies in $B_{0}(H)$.

Proof. This is the special case of [3, I Lemma 1].

Proposition 1.4 (Brauer). Let $H$ be a normal subgroup of $G$. Then for any $\tilde{\chi} \in \operatorname{Irr}\left(B_{0}(H)\right)$, there is some $\chi \in \operatorname{Irr}\left(B_{0}(G)\right)$ such that $\left(\left.\chi\right|_{H}, \tilde{\chi}\right) \neq 0$.

Proof. This is the special case of [3, II Lemma 1$]$.

Proposition 1.5 (Brauer). Let $P$ be a Sylow p-subgroup of $G$, and let $P \cdot C_{G}(P)=P \times V$. Then $k^{\prime}\left(B_{0}(G)\right)=\left|G: V G^{\prime}\right|$.

Proof. See [3, IV Proposition (4G)].

Next, we state Alperin's theorems on isomorphic principal p-blocks which are very important for our aim.

Let $F$ be an algebraically closed field of characteristic $p$ and $F G$ the group algebra of $G$ over $F$. Let $H$ be a normal subgroup of $G$ with $p \nmid|G: H|$. We write $B_{0}(G) \cong B_{0}(H)$, if the category of all finitely generated $F G$-modules in $B_{0}(G)$ is isomorphic to the category of all finitely generated $F H$-modules in $B_{0}(H)$ and if the isomorphism is given by the restriction from $G$ to $H$ (cf. [1]).

Proposition 1.6 (Alperin). Let $F$ be as above, and let $P$ be a Sylow p-subgroup of $G$. If $H$ is a normal subgroup of $G$ which satisfies the conditions that $p \nmid|G: H|, G / H$ is solvable and $G=H \cdot C_{G}(P)$, then we get the following.

(i) $B_{0}(G) \cong B_{0}(H)$.

(ii) $A_{0}(G) \cong A_{0}(H)$ as $F$-algebras, where $A_{0}(G)$ and $A_{0}(H)$ are the block ideals 
of $F G$ and $F H$ corresponding to $B_{0}(G)$ and $B_{0}(H)$, respectively.

Proof. See [1, Theorems 1 and 2].

COROLlARY 1.7 (Alperin). Let $H$ be a normal subgroup of $G$ of prime index q. with $q \neq p$. Let $B_{0}=B_{0}(G)$ and $b_{0}=B_{0}(H)$. Assume that $k\left(B_{0}\right)=k\left(b_{0}\right)$ and $\iota\left(B_{0}\right)$ $=l\left(b_{0}\right)$, and that $I_{G}(\tilde{\chi})=G$ for every $\tilde{\chi} \in \operatorname{Irr}\left(b_{0}\right)$. Then we have the following.

(i) The correspondence $\operatorname{Irr}\left(B_{0}\right) \rightarrow \operatorname{Irr}\left(b_{0}\right)$ given by $\left.\chi_{\mapsto} \rightarrow \chi\right|_{H}$ is a bijection.

(ii) The correspondence $\operatorname{IBr}\left(B_{0}\right) \rightarrow \operatorname{IBr}\left(b_{0}\right)$ given by $\left.\phi \mapsto \phi\right|_{H}$ is a bijection.

(iii) $B_{0} \cong b_{0}$.

Proof. (i) Since $I_{G}(\tilde{\chi})=G$ for every $\tilde{\chi} \in \operatorname{Irr}\left(b_{0}\right)$, the correspondence is surjective by Clifford's theorem, [8, (53.17) Theorem] and Propositions 1.3 and 1.4 . Since $k\left(B_{0}\right)=k\left(b_{0}\right)$, we obtain (i).

(ii) By (i), [1, Lemma 1] holds. Thus, by the proof of [1, Lemma 3], the correspondence is surjective. Hence (ii) holds since $l\left(B_{0}\right)=l\left(b_{0}\right)$.

(iii) Since [1, Lemmas 1 and 3] hold, we get (iii) by the proofs of Alperin's theorems [1, Theorems 1 and 2].

In the remainder of this paper we assume $p=2$ and let $G$ and $P$ be a finite group and its abelian Sylow 2-subgroup of order $2^{n}$, respectively. We use the notation $B_{0}$ and $e$ for $B_{0}(G)$ and $e(G)$, respectively.

COROllary 1.8 (Alperin). Let $H$ be a normal subgroup of $G$ of odd prime index. Let $B_{0}=B_{0}(G)$ and $b_{0}=B_{0}(H)$. Assume that $k\left(B_{0}\right)=k\left(b_{0}\right)$ and $l\left(B_{0}\right)=l\left(b_{0}\right)$, and that $H$ has an involution $x$ such that $\chi(x)= \pm 1$ for every $\chi \in \operatorname{Irr}\left(B_{0}\right)$ and $\tilde{\chi}(x)=\tilde{\chi}^{\prime}(x)= \pm 1$ for all $\tilde{\chi}, \tilde{\chi}^{\prime} \in \operatorname{Irr}\left(b_{0}\right)$ with $\tilde{\chi}(1)=\tilde{\chi}^{\prime}(1)$. Then $B_{0} \cong b_{0}$.

Proof. By Clifford's theorem and Proposition 1.3, we have $\left.\chi\right|_{H} \in \operatorname{Irr}\left(b_{0}\right)$ for all $\chi \in \operatorname{Irr}\left(B_{0}\right)$. Thus, by Proposition $1.4, I_{G}(\tilde{\chi})=G$ for all $\tilde{\chi} \in \operatorname{Irr}\left(b_{0}\right)$. Thus the corollary is proved by Corollary 1.7 (iii).

Lemma 1.9. Let $P$ be an abelian Sylow 2-subgroup of $G$. Suppose that $k\left(B_{0}\right)$ $=|P|$ and that $G$ has an involution $x$ with $l\left(b_{x}\right)=1$. Then $\chi(x)= \pm 1$ for all $\chi \in \operatorname{Irr}\left(B_{0}\right)$.

Proof. Since $l\left(b_{x}\right)=1, b_{x}$ has the unique Cartan invariant $|P|$. Hence, by [10, Theorems 63.3(2), 63.2 and 65.4], we get $\Sigma \chi(x)^{2}=|P|$ where the sum runs through all $\chi \in \operatorname{Irr}\left(B_{0}\right)$. By [4, II $(7 \mathrm{~A})$ and $\left.(4 \mathrm{C})\right], \chi(x)$ is a nonzero integer for every $\chi \in \operatorname{Irr}\left(B_{0}\right)$ since $|x|=2$. Therefore, the assumption $k\left(B_{0}\right)=|P|$ implies the 
lemma.

Proposition 1.10 (Bender, Janko, Janko-Thompson, Walter, Ward). If $G$ has abelian Sylow 2-subgroups, then $O^{\prime}(G / O(G)$ ) is a direct product of an abelian 2-group and simple groups of one of the following types;

(1) the special linear group $S L\left(2,2^{n}\right)$ for $n \geqq 2$,

(2) the projective special linear group $L_{2}(q)$ for $q>3$ with $q \equiv 3$ or $5(\bmod 8)$,

(3) the Janko's first simple group $J_{1}$,

(4) the simple group $R(q)$ of Ree type.

Proof. For groups of types (1) and (2), see [14, p. 40]. For $J_{1}$ see [16], and for $R(q)$ see [21]. The proposition is obtained from [2], [16], [17], [20] and $[21]$.

In the rest of this paper we use the notation $S L\left(2,2^{n}\right), L_{2}(q), J_{1}$ and $R(q)$ as in Proposition 1.10 (cf. [13, p. 415]). We also use the notation $G L(m, 2)$ for the general linear group (cf. [14, p. 40]).

The next lemma shows that Brauer's conjecture on heights of irreducible complex characters in $p$-blocks with abelian defect groups is affirmative for the principal 2-blocks of finite groups with abelian Sylow 2-subgroups.

LEMMA 1.11. If $G$ has abelian Sylow 2-subgroups, then all irreducible complex characters in $B_{0}(G)$ have height zero.

Proof. We may assume $O(G)=1$ by Lemma 1.1. Let $H$ be a normal subgroup of $G$ of odd index. If $\chi \in \operatorname{Irr}\left(B_{0}(G)\right)$, then there is some $\tilde{\chi} \in \operatorname{Irr}\left(B_{0}(H)\right)$ with $\chi(1)=m \tilde{\chi}(1)$ for a positive integer $m$ from Clifford's theorem and Proposition 1.3. By $[8,(53.17)$ Theorem $], m$ divides $|G: H|$. This shows that if $\tilde{\chi}(1)$ is odd then $\chi(1)$ is also odd. Thus, we may assume $O^{\prime}(G)=G$. Then, by Proposition 1.10 , we can write $G=Q \times\left(\Pi S_{i}\right)$ where $Q$ is an abelian 2-group and each $S_{i}$ is a simple group of one of the following types;

(i) $S L\left(2,2^{n}\right)$ for $n \geqq 2$,

(ii) $L_{2}(q)$ for $q>3$ with $q \equiv 3$ or $5(\bmod 8)$,

(iii) $J_{1}$,

(iv) $R(q)$.

When $S_{i}$ is of type (i) or (ii), every $\chi \in \operatorname{Irr}\left(B_{0}\left(S_{i}\right)\right)$ has odd degree from [10, Theorems 38.2 and 38.1]. When $S_{i}$ is of type (iii) or (iv), every $\chi \in \operatorname{Irr}\left(B_{0}\left(S_{i}\right)\right.$ ) has odd degree from [16, Lemma 5.1] and [21, Chap. I], respectively. These show that every $\chi \in \operatorname{Irr}\left(B_{0}(G)\right)$ has odd degree. This completes the proof. 
The next three lemmas are useful in order to obtain $e=e(G)$.

LemMA 1.12. Let $P$ be a Sylow 2-subgroup of $G$.

(i) If $G=S L\left(2,2^{n}\right)$ for $n \geqq 2$, then $P$ is elementary abelian of order $2^{n}$ and $N_{G}(P) / C_{G}(P)$ is cyclic of order $2^{n}-1$.

(ii) If $G=L_{2}(q)$ for $q>3$ with $q \equiv 3$ or $5(\bmod 8)$, then $P$ is noncyclic of order 4 and $N_{G}(P) / C_{G}(P)$ is cyclic of order 3 .

(iii) If $G=J_{1}$ or $R(q)$, then $P$ is elementary abelian of order 8 and $N_{G}(P) / C_{G}(P)$ is noncyclic of order 21 .

Proof. (i) By [14, Theorems 2.8.1 and 2.8.3], $P$ is elementary abelian of order $2^{n}$. Let $q=2^{n}$, and let $F_{q}$ be the finite field of $q$ elements. We may assume that $P=\left\{\left(\begin{array}{ll}1 & 0 \\ f & 1\end{array}\right) \mid f \in F_{q}\right\}$ (cf. the proof of [14, Theorem 2.8.3]). Clearly, $C_{G}(P)=P$. Let $u$ be a generator of the multiplicative group $F_{q}-\{0\}$, and let $s=\left(\begin{array}{ll}u & 0 \\ 0 & u^{-1}\end{array}\right)$ in $G$. Then, $N_{G}(P)=\langle P, s\rangle$ and $s$ has order $q-1$. Hence we get that $N_{G}(P) / P$ is cyclic of order $q-1$.

(ii) $P$ is noncyclic of order 4 from [14, Lemma 15.1.1]. Hence Aut $(P)$ is isomorphic to the symmetric group of degree 3. Since $G$ is not 2-nilpotent, we get (ii).

(iii) If $G=J_{1}$, we obtain (iii) from [16, VI p. 160]. Assume $G=R(q)$. By $\left[21\right.$, p. 63], $P$ is elementary abelian of order 8 and $\left|N_{G}(P): C_{G}(P)\right|=21$. Then we know that $N_{G}(P) / C_{G}(P)$ is noncyclic since $\operatorname{Aut}(P) \cong G L(3,2) \subseteq G L(4,2) \cong A_{8}$ from [15, II 2.5 Satz] where $A_{8}$ is the alternating group of degree 8 .

LEMMA 1.13. (i) $G L(4,2) \cong A_{8}$, the alternating group of degree 8 .

(ii) If $H$ is a subgroup of $A_{8}$ of odd order, then $|H|=1,3,5,7,9,15$ or 21.

(iii) $A_{8}$ has subgroups of orders $1,3,5,7,9,15$ and 21 , and the subgroups of order 9 and the subgroups of order 21 are noncyclic.

Proof. (i ) We have already showed (i) in the proof of Lemma 1.12(iii).

(ii) Since $\left|A_{8}\right|=2^{6} \cdot 3^{2} \cdot 5 \cdot 7,|H|=1,3,5,7,9,15,21,35,45,63,105$ or 315. Since the groups of order 35 are cyclic, $|H| \neq 35$. By elementary calculations, $A_{8}$ has no subgroups of order 45 , so that $|H| \neq 45$. Similarly, $|H| \neq 63$. If $|H|$ $=105$, then $H$ has an element of order 35. Evidently, this is a contradiction. Hence $|H| \neq 105$. If $|H|=315$, then $H$ has an element of order 35 , and this is a contradiction. So that $|H| \neq 315$.

(iii) By Sylow's theorem, $A_{8}$ has subgroups of orders 3, 5, 7 and 9. Since $A_{8}$ has no elements of order 9 , Sylow 3-subgroups of $A_{8}$ are noncyclic of order 
9. If $G=S L\left(2,2^{4}\right)$, then $P$ is elementary abelian of order 16 and $N_{G}(P) / C_{G}(P)$ is cyclic of order 15 from Lemma 1.12(i). Thus, by (i), $A_{8}$ has subgroups of order 15. Let $H=\langle(124)(536),(1234567)\rangle$. Then $H$ is a noncyclic subgroup of $A_{8}$ of order 21. Since $A_{8}$ has no elements of order 21, all subgroups of $A_{8}$ of order 21 are noncyclic.

Lemma 1.14. (i) If $H$ is a subgroup of $G L(3,2)$ of odd order, then $|H|=$ $1,3,7$ or 21 .

(ii) $G L(3,2)$ has subgroups of orders $1,3,7$ and 21 , and the subgroups of order 21 are noncyclic.

Proof. (i) By $[10$, Lemma $35.2(1)],|G L(3,2)|=2^{3} \cdot 3 \cdot 7$. So that we easily get (i).

(ii) By the proof of (i) and Sylow's theorem, $G L(3,2)$ has subgroups of orders 3 and 7. By Lemma 1.12(iii), $G L(3,2)$ has noncyclic subgroups of order 21. Since $G L(3,2) \subset G L(4,2)$, all subgroups of $G L(3,2)$ of order 21 are noncyclic from Lemma 1.13(i) and (iii).

The next two lemmas are useful in order to determine $B_{0}$ when Sylow 2-subgroups of $G$ are elementary abelian of order 8 or 16 .

LemmA 1.15. Let $P$ be an abelian Sylow 2-subgroup of $G$, and let $B_{0}=B_{0}(G)$. Assume that $G$ has an involution $x$ with $l\left(b_{x}\right)=1$.

(1) If $|P|=8$, then $k\left(B_{0}\right)=8$.

(2) If $|P|=16$, then $k\left(B_{0}\right)=8$ or 16 .

PROOF. Let $\left\{\chi_{1}, \cdots, \chi_{k\left(B_{0}\right)}\right\}=\operatorname{Irr}\left(B_{0}\right)$. Since $l\left(b_{x}\right)=1$, by $[10$, Theorems 63.2 and 65.4], for each $\chi_{i}$ let $d_{i 1}^{x}$ be the generalized decomposition number of $B_{0}$ relative to $x$. By Lemma 1.11 and [4, II (7A) and (4C)], every $d_{i 1}^{x}$ is an odd integer. Since $b_{x}$ has the unique Cartan invariant $|P|$, by [10, Theorem 63.3], $\sum_{i=1}^{k\left(B_{0}\right)}\left(d_{i 1}^{x}\right)^{2}=|P|$. These imply (1) and (2).

LEMMA 1.16. Let $G=L_{2}(q)$ for $q>3$ with $q \equiv 3$ or $5(\bmod 8)$, and let $B_{0}=$ $B_{0}(G)$. Then we have the following.

(i) $l\left(B_{0}\right)=3$ and the degrees of all irreducible Brauer characters in $B_{0}$ are $1,(q-1) / 2$ and $(q-1) / 2$.

(ii) The decomposition matrix of $B_{0}$ is as follows: 


$\begin{array}{rrlrll}1 & 0 & 0 & 1 & 0 & 0 \\ 0 & 1 & 0 & 1 & 1 & 0 \\ 0 & 0 & 1 & 1 & 0 & 1 \\ 1 & 1 & 1 & 1 & 1 & 1 . \\ 3<q & \equiv 3(\bmod 8) & 3<q \equiv 5(\bmod 8)\end{array}$

Proof. Since $G$ is not 2-nilpotent, $l\left(B_{0}\right)>1$ from [10, Corollary 65.3]. Thus $k\left(B_{0}\right)=4$ and $l\left(B_{0}\right)=3$ by [3, IV Proposition (7D)].

Case 1. $3<q \equiv 3(\bmod 8)$ : Let $\operatorname{Irr}\left(B_{0}\right)=\left\{\chi_{1}, \cdots, \chi_{4}\right\}$. By $[10$, Theorem 38.1], we may assume $\chi_{1}=1_{G}, \chi_{2}(1)=\chi_{3}(1)=(q-1) / 2$ and $\chi_{4}(1)=q$. By [14, Theorem 2.8.2], $G$ has a Frobenius subgroup $E$ of order $q(q-1) / 2$. We know the character tables of $E$ and $L_{2}(q)$ from [10, Theorems 13.8 and 38.1]. Thus, by [8, $\S 84$ Exercise 2], $\left.\chi_{2}\right|_{G_{0}}$ and $\left.\chi_{3}\right|_{G_{0}}$ are both irreducible Brauer characters of $G$, where $\left.\chi_{i}\right|_{G_{0}}$ is the restriction of $\chi_{i}$ to the set $G_{0}$ of all 2 '-elements of $G$. Since $\chi_{2} \neq \chi_{3}$ on $G_{0}$, and since $\chi_{4}=\chi_{1}+\chi_{2}+\chi_{3}$ on $G_{0}$, we know (i) and the decomposition matrix of $B_{0}$. Case 2. $3<q \equiv 5(\bmod 8):$ As in Case 1 we can prove the lemma.

REMARK 1. If $G$ has an abelian Sylow 2-subgroup $P$ and if $e(G)=1$, then $B_{0}(G) \cong B_{0}(P)$ since $G$ is 2 -nilpotent by [10, Theorem 18.7].

\section{The case $e=2^{m}-1$}

In this section we consider the case when $e=2^{m}-1$ for $m \geqq 2$. We use the notation $G, P, n, e$ and $B_{0}$ as before, that is to say, $P$ is an abelian Sylow 2-subgroup of $G$ with order $2^{n}(n \geqq 2), e=e(G)$ and $B_{0}=B_{0}(G)$. To begin with we state the next three lemmas which will be needed for the main result of this section.

LEMMA 2.1. Let $S$ be a normal subgroup of $G$ of odd index such that $S \cong S L\left(2,2^{n}\right)$ for some $n \geqq 3$. Assume $e=2^{n}-1$. Then $B_{0} \cong B_{0}(S)$.

Proof. We may assume $S=S L\left(2,2^{n}\right)$. There are an element $t \in N_{S}(P)$ and an involution $x \in P$ such that $N_{S}(P)=\left\langle t, C_{S}(P)\right\rangle$ and $P=\left\{1, x, x^{t}, \cdots, x^{t^{2}}-2\right\}$ (cf. the proof of Lemma 1.12(i)). Since $e=2^{n}-1, N_{G}(P)=\left\langle t, C_{G}(P)\right\rangle$. Clearly $y^{t} \neq y$ for all $y \in P-\{1\}$, so that $N_{M}(P)=C_{M}(P)$ where $M=C_{G}(x)$. Hence $M$ is 2-nilpotent from [10, Theorem 18.7]. Thus, by [10, Corollary 65.3], $l\left(b_{x}\right)=l\left(B_{0}(M)\right)$ $=1$. Now, we prove the lemma by induction on $|G|$. Suppose $G \neq S$. Since $|G / S|$ is odd, by [12, Theorem], $G$ has a normal subgroup $H$ of odd prime index $l$ with $S \subseteq H$. Let $b_{0}=B_{0}(H)$. By induction, $b_{0} \cong B_{0}(S)$. Hence, by the character table of $S L\left(2,2^{n}\right)[10$, Theorem 38.2], we get 


$\begin{array}{lcrl} & 1 & x & \\ 1_{H} & 1 & 1 & \\ \tilde{\theta}_{i} & 2^{n}-1 & -1 & \text { for } i=1, \cdots, 2^{n-1} \\ \tilde{\chi}_{j} & 2^{n}+1 & 1 & \text { for } j=1, \cdots, 2^{n-1}-1\end{array}$

where $\left\{1_{H}, \tilde{\theta}_{i}, \tilde{\chi}_{j} \mid i=1, \cdots, 2^{n-1} ; j=1, \cdots, 2^{n-1}-1\right\}=\operatorname{Irr}\left(b_{0}\right)$. Let $C_{G}(P)=P \times V$. If $G=V H$, then $G=C_{G}(P) \cdot H$, so that $B_{0} \cong b_{0}$ from Proposition 1.6. Hence we may assume $G \neq V H$. Then $H=V H$, so that $C_{H}(P)=P \times V$. Thus, by Proposition 1.5, $k^{\prime}\left(b_{0}\right)=\left|H: V H^{\prime}\right|$. Since $b_{0} \cong B_{0}(S), k^{\prime}\left(b_{0}\right)=1$. Thus, $H=V H^{\prime}$. This implies $H=V G^{\prime}$ since $G / H$ is cyclic. Hence $k^{\prime}\left(B_{0}\right)=l$ from Proposition 1.5. By Clifford's theorem and Proposition 1.3, for each $\chi \in \operatorname{Irr}\left(B_{0}\right)$ one of the following five cases occurs :

(a) $\left.\chi\right|_{H}=1_{H}$,

(b) $\left.\chi\right|_{H}=\tilde{\theta}_{i}$ for some $i$,

(c) $\left.\chi\right|_{H}=\tilde{\theta}_{i_{1}}+\cdots+\tilde{\theta}_{i_{l}}$ for $i_{1}<\cdots<i_{l}$, and all $\tilde{\theta}_{i_{k}}$ are $G$-conjugate,

(d) $\left.\chi\right|_{H}=\tilde{\chi}_{j}$ for some $j$,

(e) $\left.\chi\right|_{H}=\tilde{\chi}_{j_{1}}+\cdots+\tilde{\chi}_{j_{l}}$ for $j_{1}<\cdots j_{l}$, and all $\tilde{\chi}_{j_{k}}$ are $G$-conjugate.

Since $k^{\prime}\left(b_{0}\right)=1$, for each $\chi \in \operatorname{Irr}\left(B_{0}\right) \chi(1)=1$ if and only if $\left.\chi\right|_{H}=1_{H}$. Let $r, s, u$ and $v$ be the numbers of $\chi \in \operatorname{Irr}\left(B_{0}\right)$ of types (b), (c), (d) and (e), respectively. Since $l\left(b_{x}\right)=1$, as in the proof of Lemma $1.9, \Sigma \chi(x)^{2}=2^{n}$ where the sum runs through all $\chi \in \operatorname{Irr}\left(B_{0}\right)$. This shows $l+r+s l^{2}+u+v l^{2}=2^{n}$. On the other hand, by Proposition 1.4, for every $\tilde{\chi} \in \operatorname{Irr}\left(b_{0}\right)$ there is some $\chi \in \operatorname{Irr}\left(B_{0}\right)$ with $\left(\left.\chi\right|_{H}, \tilde{\chi}\right) \neq 0$. So that $k\left(b_{0}\right) \leqq 1+r+s l+u+v l$. Since $k\left(b_{0}\right)=2^{n}$, we have a contradiction. This completes the proof.

REMARK 1. We can not remove the assumption $e=2^{n}-1$ in Lemma 2.1. Indeed, let $S=S L(2,8)$ and $P=\left\{\left(\begin{array}{ll}1 & 0 \\ f & 1\end{array}\right) \mid f \in F_{8}\right\}$ where $F_{8}$ is the finite field of 8 elements. Let $u$ be a generator of the multiplicative group $F_{8}-\{0\}$. There is an automorphism $h$ of $F_{8}$ with $h(u)=u^{2}$. For each $\left(\begin{array}{ll}a & b \\ c & d\end{array}\right) \in S \operatorname{let}\left(\begin{array}{ll}a & b \\ c & d\end{array}\right)^{h}=$ $\left(\begin{array}{ll}h(a) & h(b) \\ h(c) & h(d)\end{array}\right)$. Then we can consider $h \in \operatorname{Aut}(S)$ and $\left.h\right|_{P} \in \operatorname{Aut}(P)$ where $\left.h\right|_{P}$ is the restriction of $h$ to $P$. Hence there is a semi-direct product $G$ of its normal subgroup $S$ by $\langle h\rangle$. Then $O^{\prime}(G)=S=S L(2,8)$ and $e(G)=21 \neq 2^{3}-1$. By [10, Theorem 38.2], $l\left(B_{0}(S)\right)=7$. But we shall afterwards show that $l\left(B_{0}(G)\right)=5$, and this shows $B_{0}(G) \cong B_{0}(S)$.

LEMMA 2.2. Let $S$ be a normal subgroup of $G$ of odd index such that $S \cong L_{2}(q) \times\left(P /\left(Z_{2} \times Z_{2}\right)\right)$ for some $q>3$ with $q \equiv 3$ or $5(\bmod 8)$, or $S \cong S L\left(2,2^{m}\right)$ 
$\times\left(P /(\underbrace{Z_{2} \times \cdots \times Z_{2}}_{m})\right)$ for some $m \geqq 3$. Assume $e=e(S)$. Then $k\left(B_{0}\right)=2^{n}$ and $l\left(B_{0}\right)=e$.

Proof. Let $L=L_{2}(q)$ for $m=2$, and let $L=S L\left(2,2^{m}\right)$ for $m \geqq 3$. Let $R$ be a Sylow 2-subgroup of $L$. We can write $S=L \times Q$ and $P=R \times Q$. We use induction on $n$. If $n=m=2$, then the lemma is proved by [3, IV Proposition (7D)]. If $n=m \geqq 3$, by Lemma $2.1, B_{0} \cong B_{0}(S)$, so that $k\left(B_{0}\right)=2^{n}$ and $l\left(B_{0}\right)=2^{n}-1$ $=2^{m}-1$ (cf. [10, Theorem 38.2]). Next, suppose $n>m$. There are an element $t \in N_{L}(R)$ and an involution $x \in R$ such that $N_{L}(R)=\left\langle t, C_{L}(R)\right\rangle$ and $R=\left\{1, x, x^{t}\right.$, $\left.\cdots x^{t^{2^{m}-2}}\right\}$. Since $e=e(S), N_{G}(P)=\left\langle t, C_{G}(P)\right\rangle$. Let $Q=\left\{1=y_{1}, y_{2}, \cdots, y_{2^{n-m}}\right\}$. Then, by [10, Lemma 18.5], the $G$-conjugate classes of $P$ are as follows:

$$
\begin{gathered}
\{1\} \\
\left\{y_{i}\right\} \text { for } i=2, \cdots, 2^{n-m} \\
\left\{x y_{i}, x^{t} y_{i}, \cdots, x^{t^{2^{m}-2}} y_{i}\right\} \text { for } i=1, \cdots, 2^{n-m} .
\end{gathered}
$$

Then, by [10, Theorems 68.4 and 65.4],

$$
k\left(B_{0}\right)=l\left(B_{0}\right)+\sum_{i=2}^{2 n-m} l\left(b_{y_{i}}\right)+\sum_{i=1}^{2 n-m} l\left(b_{x y_{i}}\right) .
$$

Fix any $i$ with $2 \leqq i \leqq 2^{n-m}$, and let $M=C_{G}\left(y_{i}\right)$. Since $y_{i} \in Z(S)$, let $\bar{S}=S /\left\langle y_{i}\right\rangle$. Similarly, let $\bar{M}=M /\left\langle y_{i}\right\rangle, \bar{P}=P /\left\langle y_{i}\right\rangle$ and $\bar{Q}=Q /\left\langle y_{i}\right\rangle$. Since $\bar{S} \cong L \times \bar{Q}$, we get $e(\bar{S})=e(L)=2^{m}-1$. Since $\bar{S} \cong \bar{M}$, the canonical homomorphism $N_{\bar{S}}(\bar{P}) / C_{\bar{S}}(\bar{P}) \rightarrow$ $N_{\bar{M}}(\bar{P}) / C_{\bar{M}}(\bar{P})$ is monomorphic. This shows $\left(2^{m}-1\right) \mid e(\bar{M})$. On the other hand, by $\left[15\right.$, I 7.7 Hilfssatz (c)], we get $N_{\bar{M}}(\bar{P})=\left(N_{M}(P) \cdot\left\langle y_{i}\right\rangle\right) /\left\langle y_{i}\right\rangle$. This implies that the canonical homomorphism $N_{M}(P) / C_{M}(P) \rightarrow N_{\bar{M}}(\bar{P}) / C_{\bar{M}}(\bar{P})$ is epimorphic. Hence $e(\bar{M}) \mid e(M)$. Since $S \subseteq M \cong G$ and $e=e(S)=2^{m}-1$, we have $e(M)=e(S)=2^{m}-1$ by considering the canonical monomorphisms as above. Thus $e(\bar{M})=2^{m}-1$. Hence we get $l\left(B_{0}(\bar{M})\right)=2^{m}-1$ by induction. Thus $l\left(b_{y_{i}}\right)=l\left(B_{0}(M)\right)=2^{m}-1$ from Proposition 1.2. We may assume $O(G)=1$ by Lemma 1.1. Since $Q \neq 1$, there is an involution $y_{j} \in Q$. By $Z^{*}$-theorem [10, Theorem 67.1], $y_{j} \in Z(G)$. Hence $l\left(B_{0}\right)$ $=l\left(b_{y_{j}}\right)=2^{m}-1$. Next, we consider $l\left(b_{x y_{i}}\right)$ for each $i=1, \cdots, 2^{n-m}$. For an integer $k$ it is seen that $\left(x y_{i}\right)^{t^{k}}=x y_{i}$ if and only if $\left(2^{m}-1\right) \mid k$. Hence $N_{U}(P)=C_{U}(P)$ where $U=C_{G}\left(x y_{i}\right)$. Then $U$ is 2-nilpotent from [10, Theorem 18.7], so that $l\left(b_{x y_{i}}\right)=l\left(B_{0}(U)\right)=1$ by $\left[10\right.$, Corollary 65.3]. These imply $k\left(B_{0}\right)=2^{n}$.

LEMmA 2.3. Assume as in Lemma 2.2. Then $B_{0} \cong B_{0}(S)$.

Proof. We use the same notation as in the proof of Lemma 2.2. We prove the lemma by induction on $|G|$. Suppose $G \neq S$. By [12, Theorem], $G$ has a normal subgroup $H$ of odd prime index with $S \subseteq H$. Let $b_{0}=B_{0}(H)$. By induction, $b_{0} \cong B_{0}(S)$. It follows from Lemma 2.2 that $k\left(B_{0}\right)=k\left(b_{0}\right)=2^{n}$ and that 
$l\left(B_{0}\right)=l\left(b_{0}\right)=2^{m}-1$. By the proof of Lemma 2.2, there is an involution $x \in G$ with $l\left(b_{x}\right)=1$. Hence $\chi(x)= \pm 1$ for all $\chi \in \operatorname{Irr}\left(B_{0}\right)$ from Lemma 1.9. Thus, by Corollary 1.8 , it is sufficient to show that

$$
\begin{aligned}
& \text { if } \tilde{\chi}, \tilde{\chi}^{\prime} \in \operatorname{Irr}\left(b_{0}\right) \text { with } \tilde{\chi}(1)=\tilde{\chi}^{\prime}(1), \\
& \text { then } \tilde{\chi}(x)=\tilde{\chi}^{\prime}(x)= \pm 1
\end{aligned}
$$

Let $\left\{\theta_{1}, \cdots, \theta_{2 n-m}\right\}$ be the set of all irreducible complex characters of $Q$.

Case 1. $m=2$ : By the character table of $L_{2}(q)$ (cf. [10, Theorem 38.1]), we can write

$$
\begin{array}{lcc} 
& 1 & x \\
\zeta_{1} & 1 & 1 \\
\zeta_{2} & (q+\varepsilon) / 2 & -\varepsilon \\
\zeta_{3} & (q+\varepsilon) / 2 & -\varepsilon \\
\zeta_{4} & q & \varepsilon,
\end{array} \quad \varepsilon=\left\{\begin{array}{r}
-1 \text { if } q \equiv 3(\bmod 8) \\
1 \text { if } q \equiv 5(\bmod 8)
\end{array}\right.
$$

where $\left\{\zeta_{1}, \cdots, \zeta_{4}\right\}=\operatorname{Irr}\left(B_{0}\left(L_{2}(q)\right)\right)$. Since $b_{0} \cong B_{0}(S)$ and since $S=L_{2}(q) \times Q$, we may write $\operatorname{Irr}\left(b_{0}\right)=\left\{\tilde{\chi}_{i j} \mid i=1, \cdots, 4 ; j=1, \cdots, 2^{n-2}\right\}$ such that $\left.\tilde{\chi}_{i j}\right|_{S}=\zeta_{i} \theta_{j}$ for all $i, j$. Then

$$
\tilde{\chi}_{i j}(1)=\left\{\begin{array}{cl}
1 & \text { for } i=1 \\
(q+\varepsilon) / 2 & \text { for } i=2,3 \\
q & \text { for } i=4
\end{array}\right.
$$

and

$$
\tilde{\chi}_{i j}(x)=\left\{\begin{aligned}
1 & \text { for } i=1 \\
-\varepsilon & \text { for } i=2,3 \\
\varepsilon & \text { for } i=4 .
\end{aligned}\right.
$$

These imply $(*)$.

Case 2. $m \geqq 3$ : By the character table of $S L\left(2,2^{m}\right)$ (cf. [10, Theorem 38.2]), we know

$$
\begin{array}{lcrl} 
& 1 & x & \\
1 & 1 & 1 & \\
\tilde{\theta}_{i} & 2^{m}-1 & -1 & \text { for } i=1, \cdots, 2^{m-1} \\
\tilde{\chi}_{j} & 2^{m}+1 & 1 & \text { for } j=1, \cdots, 2^{m-1}-1
\end{array}
$$

where $\left\{1, \tilde{\theta}_{i}, \tilde{\chi}_{j} \mid i=1, \cdots, 2^{m-1} ; j=1, \cdots, 2^{m-1}-1\right\}=\operatorname{Irr}\left(B_{0}\left(S L\left(2,2^{m}\right)\right)\right)$. Using this we can show $\left(^{*}\right)$ as in Case 1 . This completes the proof.

Now, the above lemmas imply the next main result of this section. 
THEOREM 2.4. Let $P$ be an abelian Sylow 2-subgroup of $G$. Assume that $e$ is prime. Then we have the following.

(1) $l\left(B_{0}\right)=e$. And if $G$ is nonsolvable. then $k\left(B_{0}\right)=|P|$.

(2) When $G$ is nonsolvable, one of the following holds:

(i) $e=3$, and $B_{0} \cong B_{0}\left(L_{2}(q) \times\left(P /\left(Z_{2} \times Z_{2}\right)\right)\right)$ for some $q>3$ with $q \equiv 3$ or 5 $(\bmod 8)$

(ii) $e=2^{m}-1$ for some $m \geqq 3$, and $B_{0} \cong B_{0}\left(S L\left(2,2^{m}\right) \times\left(P /(\underbrace{Z_{2} \times \cdots \times Z_{2}}_{m})\right)\right)$.

Proof. We can assume $O(G)=1$ by Lemma 1.1. Let $S=O^{\prime}(G)$. Firstly assume that $S$ is solvable. Then $S=P$, so that $C_{G}(P)=P$. Hence $G$ is a semidirect product of its normal subgroup $P$ by $Z_{e}$. This shows $l\left(B_{0}\right)=e$. So it is enough to consider the case where $G$ is nonsolvable. Since $e$ is prime, $e=e(S)$. By Proposition 1.10 and Lemma 1.12, one of the following two cases occurs:

(i) $e(S)=3$, and $S \cong L_{2}(q) \times\left(P /\left(Z_{2} \times Z_{2}\right)\right)$ for some $q>3$ with $q \equiv 3$ or $5(\bmod 8)$,

(ii) $e(S)=2^{m}-1$ for some $m \geqq 3$, and $S \cong S L\left(2,2^{m}\right) \times\left(P /(\underbrace{Z_{2} \times \cdots \times Z_{2}}_{m})\right)$.

Hence we obtain (1) and (2) from Lemmas 2.2 and 2.3, respectively.

REMARK 2. For the case where $G$ is solvable, the latter half of Theorem 2.4(1) does not hold in general. Indeed, let $P$ be an elementary abelian group of order 16 with $P=\langle x, y, z, w\rangle$. Let $t \in \operatorname{Aut}(P)$ such that $x^{t}=y, y^{t}=x y, z^{t}=w$ and $w^{t}=z w$. There is a semi-direct product $G$ of its normal subgroup $P$ by $\langle t\rangle$. Then $G$ is solvable and $e=|G: P|=3$. Since $u^{t} \neq u$ for all $u \in P-\{1\}$, we shall show that $k\left(B_{0}\right)=8 \neq 16$ (cf. Proposition 6.1). As another example, let $P$ be the same as above, and let $t \in \operatorname{Aut}(P)$ with $|t|=5$. If $G$ is a semi-direct product of $P$ by $\langle t\rangle$ and $G$ is not the direct product $P \times Z_{5}$, then we shall show that $k\left(B_{0}\right)=8 \neq 16$ (cf. Proposition 6.3).

\section{The case $e=9$}

In this section we consider the case when $e=e(S)=9$, where $S=O^{\prime}(G / O(G))$. We use the notation $G, P, n, e$ and $B_{0}$ as in $\S 2$.

LEMMA 3.1. Let $P$ be an elementary abelian Sylow 2-subgroup of $G$ of order 16. If $e=9$, then $k\left(B_{0}\right)=16$ and $l\left(B_{0}\right)=9$.

Proof. By Lemma 1.13, Aut $(P)$ has noncyclic Sylow 3-subgroups of order 9. Hence we may assume that $N_{G}(P)=\left\langle s, t, C_{G}(P)\right\rangle$ for some $s, t \in N_{G}(P), P=$ $\langle x, y, z, w\rangle, x^{s}=x, y^{s}=y, z^{s}=w, w^{s}=z w, x^{t}=y, y^{t}=x y, z^{t}=z$ and $w^{t}=w$. By [10, Lemma 18.5 and Theorems 68.4 and 65.4], 


$$
k\left(B_{0}\right)=l\left(B_{0}\right)+l\left(b_{x}\right)+l\left(b_{z}\right)+l\left(b_{x z}\right) .
$$

Since $e\left(C_{G}(x z)\right)=1, l\left(b_{x z}\right)=1$ from [10, Theorem 18.7 and Corollary 65.3]. Since $e\left(C_{G}(x)\right)=e\left(C_{G}(z)\right)=3$, it follows from Theorem 2.4 that $l\left(b_{x}\right)=l\left(b_{z}\right)=3$. By $[10$, Corollary 65.3], $l\left(B_{0}\right) \geqq 2$ since $e=9$. Hence, by Lemma $1.15(2), k\left(B_{0}\right)=16$, so that $l\left(B_{0}\right)=9$.

LEMMA 3.2. Let $S$ be a normal subgroup of $G$ of odd index such that $S \cong L_{2}(q) \times L_{2}\left(q^{\prime}\right) \times\left(P /\left(Z_{2} \times Z_{2} \times Z_{2} \times Z_{2}\right)\right)$ for some $q, q^{\prime}>3$ with $q \equiv 3$ or $5(\bmod 8)$ and $q^{\prime} \equiv 3$ or $5(\bmod 8)$. If $e=9$, then $k\left(B_{0}\right)=2^{n}$ and $l\left(B_{0}\right)=9$.

Proof. We may assume $S=L_{2}(q) \times L_{2}\left(q^{\prime}\right) \times Q$ where $Q \cong P /\left(Z_{2} \times Z_{2} \times Z_{2} \times Z_{2}\right)$. We use induction on $n$. If $n=4$, Sylow 2-subgroups of $G$ are elementary abelian of order 16, so that the lemma is proved by Lemma 3.1. Suppose $n>4$. Let $R_{1}$ and $R_{2}$ be Sylow 2-subgroups of $L_{2}(q)$ and $L_{2}\left(q^{\prime}\right)$, respectively. We may assume $P=R_{1} \times R_{2} \times Q$. We can write $R_{1}=\left\{1, x, x^{s}, x^{s^{2}}\right\}$ for some $s \in L_{2}(q)$ and for an involution $x \in R_{1}$. Similarly, $R_{2}=\left\{1, y, y^{t}, y^{t^{2}}\right\}$ for some $t \in L_{2}\left(q^{\prime}\right)$ and for an involution $y \in R_{2}$. Since $e=e(S)=9$, we know that $N_{G}(P)=\left\langle s, t, C_{G}(P)\right\rangle$ and that $N_{G}(P) / C_{G}(P)$ is elementary abelian of order 9 . Let $Q=\left\{1=z_{1}, z_{2}, \cdots\right.$, $\left.z_{2^{n-4}}\right\}$. By [10, Lemma 18.5], $\left\{z_{i}, x z_{i}, y z_{i}, x y z_{i} \mid i=1, \cdots, 2^{n-4}\right\}$ is the set of all representatives of $G$-conjugate classes of $P$. Thus, by $[10$, Theorems 68.4 and $65.4]$,

$$
\begin{aligned}
k\left(B_{0}\right)= & l\left(B_{0}\right)+\sum_{i=2}^{2^{n-4}} l\left(b_{z_{i}}\right) \\
& +\sum_{i=1}^{2^{n-4}}\left\{l\left(b_{x z_{i}}\right)+l\left(b_{y z_{i}}\right)+l\left(b_{x y z_{i}}\right)\right\} .
\end{aligned}
$$

As in the proof of Lemma 2.2, by induction, we get $l\left(b_{z_{i}}\right)=9$ for all $i=2, \cdots, 2^{n-4}$. By Lemma 1.1, we may assume $O(G)=1$. Since $Q \neq 1$, as in the proof of Lemma 2.2 , by making use of $Z^{*}$-theorem [10, Theorem 67.1], we have $l\left(B_{0}\right)=9$. Since $s \notin C_{G}\left(x z_{i}\right)$ and since $t \in C_{G}\left(x z_{i}\right)$, we obtain $e\left(C_{G}\left(x z_{i}\right)\right)=3$. Hence $l\left(b_{x z_{i}}\right)=3$ for all $i=1, \cdots, 2^{n-4}$ from Theorem 2.4(1). Similarly, by Theorem 2.4(1), $l\left(b_{y z_{i}}\right)=3$ for all $i=1, \cdots, 2^{n-4}$. Fix any $i$ with $1 \leqq i \leqq 2^{n-4}$. For integers $j$ and $k$, it is seen that $\left(x y z_{i}\right)^{s^{j} t^{k}}=x y z_{i}$ if and only if $3 \mid j$ and $3 \mid k$. Hence as in the proof of Lemma $2.2, l\left(b_{x y z_{i}}\right)=1$ for all $i=1, \cdots, 2^{n-4}$. Thus $k\left(B_{0}\right)=2^{n}$. This finishes the proof.

Lemma 3.3. Assume as in Lemma 3.2. Then $B_{0} \cong B_{0}(S)$.

Proof. We use the same notation as in the proof of Lemma 3.2. We prove the lemma by induction on $|G|$. Assume $G \neq S$. By [12, Theorem], $G$ has a normal subgroup $H$ of odd prime index with $S \subseteq H$. Let $b_{0}=B_{0}(H)$. By 
induction, $b_{0} \cong B_{0}(S)$. By the proof of Lemma 3.2, there is an involution $x y \in G$ with $l\left(b_{x y}\right)=1$. It follows from Lemmas 3.2 and 1.9 that $\chi(x y)= \pm 1$ for all $\chi \in \operatorname{Irr}\left(B_{0}\right)$. By Lemma 3.2, $k\left(B_{0}\right)=k\left(b_{0}\right)$ and $l\left(B_{0}\right)=l\left(b_{0}\right)$. Thus, by Corollary 1.8, it is enough to prove that

if $\tilde{\chi}, \tilde{\chi}^{\prime} \in \operatorname{Irr}\left(b_{0}\right)$ with $\tilde{\chi}(1)=\tilde{\chi}^{\prime}(1)$,

then $\tilde{\chi}(x y)=\tilde{\chi}^{\prime}(x y)= \pm 1$.

As in the proof of Lemma 2.3 we know the character tables of $L_{2}(q)$ and $L_{2}\left(q^{\prime}\right)$. Thus we can write

$$
\begin{array}{lcc} 
& 1 & x \\
\eta_{1} & 1 & 1 \\
\eta_{2} & (q+\varepsilon) / 2 & -\varepsilon \\
\eta_{3} & (q+\varepsilon) / 2 & -\varepsilon \\
\eta_{4} & q & \varepsilon
\end{array} \quad \varepsilon=\left\{\begin{aligned}
-1 & \text { if } q \equiv 3(\bmod 8) \\
1 & \text { if } q \equiv 5(\bmod 8)
\end{aligned}\right.
$$

where $\left\{\eta_{1}, \eta_{2}, \eta_{3}, \eta_{4}\right\}=\operatorname{Irr}\left(B_{0}\left(L_{2}(q)\right)\right)$, and

$$
\begin{array}{lcc} 
& 1 & y \\
\zeta_{1} & 1 & 1 \\
\zeta_{2} & \left(q^{\prime}+\varepsilon^{\prime}\right) / 2 & -\varepsilon^{\prime} \\
\zeta_{3} & \left(q^{\prime}+\varepsilon^{\prime}\right) / 2 & -\varepsilon^{\prime} \\
\zeta_{4} & q^{\prime} & \varepsilon^{\prime}
\end{array} \quad \varepsilon^{\prime}=\left\{\begin{aligned}
-1 & \text { if } q^{\prime} \equiv 3(\bmod 8) \\
1 & \text { if } q^{\prime} \equiv 5(\bmod 8)
\end{aligned}\right.
$$

where $\left\{\zeta_{1}, \zeta_{2}, \zeta_{3}, \zeta_{4}\right\}=\operatorname{Irr}\left(B_{0}\left(L_{2}\left(q^{\prime}\right)\right)\right)$. Let $\left\{\theta_{1}, \cdots \theta_{2 n-4}\right\}$ be the set of all irreducible complex characters of $Q$. Since $b_{0} \cong B_{0}(S)$, we may write $\operatorname{Irr}\left(b_{0}\right)=\left\{\tilde{\chi}_{i j k} \mid i=1, \cdots, 4\right.$; $\left.j=1, \cdots, 4 ; k=1, \cdots, 2^{n-4}\right\}$ such that $\left.\tilde{\chi}_{i j k}\right|_{s}=\eta_{i} \zeta_{j} \theta_{k}$ for all $i, j, k$.

Case 1. $\varepsilon=-1$ and $\varepsilon^{\prime}=1$ : In order to show $\left(^{*}\right)$ it is enough to prove that $\left\{1,(q-1) / 2, q^{\prime},(q-1) q^{\prime} / 2, q\left(q^{\prime}+1\right) / 2\right\} \cap\left\{\left(q^{\prime}+1\right) / 2, q,(q-1)\left(q^{\prime}+1\right) / 4, q q^{\prime}\right\}=\varnothing \quad$ since $\tilde{\chi}_{i j k}(1)=\eta_{i}(1) \zeta_{j}(1)$ and $\tilde{\chi}_{i j k}(x y)=\eta_{i}(x) \zeta_{j}(y)$ for all $i, j, k$. We can prove it.

Case 2. $\varepsilon=\varepsilon^{\prime}=-1$ : We know that $\left\{1,(q-1) / 2,\left(q^{\prime}-1\right) / 2,(q-1)\left(q^{\prime}-1\right) / 4, q q^{\prime}\right\}$ $\cap\left\{q, q^{\prime},(q-1) q^{\prime} / 2, q\left(q^{\prime}-1\right) / 2\right\}=\varnothing$. This implies $\left(^{*}\right)$ as in Case 1 .

Case 3. $\varepsilon=\varepsilon^{\prime}=1$ : Since $\left\{1, q, q^{\prime},(q+1)\left(q^{\prime}+1\right) / 4, q q^{\prime}\right\} \cap\left\{(q+1) / 2,\left(q^{\prime}+1\right) / 2\right.$, $\left.(q+1) q^{\prime} / 2, q\left(q^{\prime}+1\right) / 2\right\}=\varnothing$, we can show $\left(^{*}\right)$. This completes the proof of the lemma.

The above lemmas imply the next main result of this section.

TheOREM 3.4. Let $P$ be an abelian Sylow 2-subgroup of G. Assume $e=e(S)$ 
$=9$, where $S=O^{\prime}(G / O(G))$. Then we have the following.

(1) $k\left(B_{0}\right)=|P|$ and $l\left(B_{0}\right)=9$.

(2) $B_{0} \cong B_{0}\left(L_{2}(q) \times L_{2}\left(q^{\prime}\right) \times\left(P /\left(Z_{2} \times Z_{2} \times Z_{2} \times Z_{2}\right)\right)\right)$ for some $q, q^{\prime}>3$ with $q \equiv 3$ or $5(\bmod 8)$ and $q^{\prime} \equiv 3$ or $5(\bmod 8)$.

Rroof. We may assume $O(G)=1$ by Lemma 1.1. Since $e(S)=9$, by Proposition 1.10 and Lemma 1.12 , we get that $S \cong L_{2}(q) \times L_{2}\left(q^{\prime}\right) \times\left(P /\left(Z_{2} \times Z_{2} \times Z_{2} \times Z_{2}\right)\right)$ for some $q, q^{\prime}>3$ with $q \equiv 3$ or $5(\bmod 8)$ and $q^{\prime} \equiv 3$ or $5(\bmod 8)$. Hence we obtain (1) and (2) from Lemmas 3.2 and 3.3, respectively.

\section{The case $e=21$}

In this section we deal with the case when $e=e(S)=21$, where $S=O^{\prime}(G / O(G))$. As in $\S 1$, let $J_{1}$ and $R(q)$ be the Janko's first simple group and the simple groups of Ree type, respectively (cf. [16], [21] and [13]). We use the notation $G, P, n, e$ and $B_{0}$ as before.

LEMMA 4.1. Let $P$ be an elementary abelian Sylow 2-subgroup of $G$ of order 8. If $e=21$, then $k\left(B_{0}\right)=8$ and $l\left(B_{0}\right)=5$.

Proof. By Lemma 1.14, $N_{G}(P) / C_{G}(P)$ is noncyclic of order 21. Hence we can write that $N_{G}(P)=\left\langle s, t, C_{G}(P)\right\rangle, P=\left\{1, x, x^{s}, x^{s^{2}}, z, x z, x^{s} z, x^{s^{2}} z\right\}=\left\{1, z, z^{t}\right.$, $\left.\cdots, z^{t^{6}}\right\}$ for some $s, t \in N_{G}(P)$ and involutions $x, z \in P$ with $z^{s}=z$. Then, by $[10$, Theorems 68.4 and 65.4], $k\left(B_{0}\right)=l\left(B_{0}\right)+l\left(b_{z}\right)$. Since $e\left(C_{G}(z)\right)=3, l\left(b_{z}\right)=3$ from Theorem 2.4(1). The calculation of the generalized decomposition matrix of $B_{0}$ relative to $z$ is due to J. B. Olsson [18, Theorems 3.15, 3.16 and 3.17]. Let $M=C_{G}(z), \bar{M}=M /\langle z\rangle$ and $\bar{b}_{z}=B_{0}(\bar{M})$. By [10, Theorem 66.3], there is a basic set $\bar{W}$ of $\bar{b}_{z}$ such that $\bar{W}$ contains the trivial Brauer character and the Cartan matrix of $\bar{b}_{z}$ with respect to $\bar{W}$ has the form

$$
\begin{array}{lll}
2 & 1 & 1 \\
1 & 2 & 1 \\
1 & 1 & 2
\end{array} .
$$

Then, by [10, Lemma 66.1], there is a basic set $W$ of $b_{z}$ such that $W$ contains the trivial Brauer character and the Cartan matrix $C_{z}$ of $b_{z}$ with respect to $W$ has the form

$$
\begin{array}{lll}
4 & 2 & 2 \\
2 & 4 & 2 \\
2 & 2 & 4 .
\end{array}
$$


We use the following notation here. For an integer $r \geqq 0$ and a $p$-block $B$, let $E_{B}\left(p^{r}\right)$ denote the multiplicity of $p^{r}$ as an elementary divisor of the Cartan matrix of $B$. If $Q$ is a $p$-subgroup of a finite group $A$ and if $B$ is a $p$-block of $A$, let $n_{B}(Q)$ denote the multiplicity of $Q$ as a lower defect group of $B$ (cf. [5]. In [5], $n_{B}(Q)$ is denoted by $\left.m_{B}^{(1)}(Q)\right)$. By [8, (89.8) Theorem], $E_{B_{0}}(8)=1$. Since all involutions in $G$ are conjugate, by [5, (7G)], [18, Proposition 1.2] and [10, Theorem 65.4], we get $E_{B_{0}}(2)=n_{b_{z}}(\langle z\rangle)$. Since every lower defect group of a 2-block of $G$ contains all 2-subgroups $U$ of $G$ with $U \subseteq Z(G)$, by [5, (7G)], $E_{b_{z}}(2)=n_{b_{z}}(\langle z\rangle)$. By $(*), E_{b_{z}}(2)=2$. Thus $E_{B_{0}}(2)=2$, so that $l\left(B_{0}\right) \geqq 3$. This shows $k\left(B_{0}\right) \geqq 6$. Let $\left\{\chi_{i} \mid i=1, \cdots, k\left(B_{0}\right)\right\}=\operatorname{Irr}\left(B_{0}\right)$. Since $l\left(b_{z}\right)=3$, let $N=\left(n_{i \alpha}\right)_{1 \leq i \leq k\left(B_{0}\right)}$ be the matrix of the generalized decomposition numbers of $B_{0}$ relative to $z$ with respect to $W$. Since $|z|=2$, every $n_{i \alpha}$ is an integer. By [4, II (7A) and (4C)], $\left(n_{i 1}, n_{i 2}, n_{i 3}\right) \neq(0,0,0)$ for every $\chi_{i}$. For $\chi_{i}, \chi_{j}$ let $a_{i j}=\sum_{1 \leq \alpha, \beta \leq 3} 8 n_{i \alpha} u_{\alpha \beta} n_{j \beta}$, where $C_{z}^{-1}=\left(u_{\alpha \beta}\right)_{1 \leq \alpha, \beta \leq 3}$. By Lemma 1.11 and $\left[4\right.$, II (7A) and (5G)], all $a_{i i}$ are odd integers. Hence $n_{i 1}+n_{i 2}+n_{i 3}$ is odd for every $\chi_{i}$. Let $N_{\alpha}$ be the $\alpha$-th column of $N$ for each $\alpha$, and let $N_{\alpha} N_{\beta}=\sum_{i=1}^{k\left(B_{0}\right)} n_{i \alpha} n_{i \beta}$ for all $\alpha, \beta$. By [10, Theorem 63.3(2)], ${ }^{t} N N=C_{z}$ where ${ }^{t} N$ is the transposed matrix of $N$. So $N_{\alpha} N_{\beta}$ $=4$ if $\alpha=\beta$, and $N_{\alpha} N_{\beta}=2$ if $\alpha \neq \beta$. Clearly, $12=\operatorname{tr}\left(C_{z}\right)=\sum_{i, \alpha} n_{i \alpha}{ }^{2}$ where $\operatorname{tr}\left(C_{z}\right)$ is the trace of $C_{z}$. Then the next three possibilities arise for the nonzero entries of $N$ :

(i) 2 entries are \pm 2 , and 4 entries are \pm 1 .

(ii) 1 entry is \pm 2 , and 8 entries are \pm 1 .

(iii) 12 entries are \pm 1 .

By elementary calculations as in [18, Theorems $3.15,3.16$ and 3.17$]$ we can write

$$
\begin{array}{rll}
\delta_{1} & 0 & 0 \\
\delta_{2} & 0 & 0 \\
0 & \delta_{3} & 0 \\
0 & \delta_{4} & 0 \\
0 & 0 & \delta_{5} \\
0 & 0 & \delta_{6} \\
\delta_{7} & \delta_{7} & \delta_{7} \\
\delta_{8} & \delta_{8} & \delta_{8}
\end{array}
$$

where $\delta_{i}= \pm 1$. This shows $k\left(B_{0}\right)=8$, so that $l\left(B_{0}\right)=5$. This completes the proof.

LEMMA 4.2. Let $S$ be a normal subgroup of $G$ of odd index such that $S \cong J_{1} \times\left(P /\left(Z_{2} \times Z_{2} \times Z_{2}\right)\right)$ or $S \cong R(q) \times\left(P /\left(Z_{2} \times Z_{2} \times Z_{2}\right)\right)$. If $e=21$, then $k\left(B_{0}\right)=2^{n}$ and $l\left(B_{0}\right)=5$. 
Proof. We may assume $S=R \times Q$ where $R=J_{1}$ or $R(q)$ and $Q \cong P /\left(Z_{2} \times Z_{2}\right.$ $\times Z_{2}$ ). Let $T$ be a Sylow 2-subgroup of $R$ with $T \times Q=P$. By Lemma 1.12(iii), $N_{R}(T) / C_{R}(T)$ is noncyclic of order 21 . Hence we can write $N_{R}(T)=\left\langle s, t, C_{R}(T)\right\rangle$ and $T=\left\{1, x, x^{s}, x^{s^{2}}, z, x z, x^{s} z, x^{s^{2}} z\right\}=\left\{1, x, x^{t}, \cdots, x^{t^{6}}\right\}$ for some $s, t \in N_{R}(T)$ and for involutions $x, z \in T$ with $z^{s}=z$. Since $e=21, N_{G}(P)=\left\langle s, t, C_{G}(P)\right\rangle$. We prove the lemma by induction on $n$. If $n=3$, the lemma is proved from Lemma 4.1 because $P=T$ and $P$ is elementary abelian of order 8 from Lemma 1.12(iii). Suppose $n>3$. Let $Q=\left\{1=y_{1}, y_{2}, \cdots, y_{2 n-3}\right\}$. By [10, Lemma 18.5], $\left\{y_{i}, z y_{i} \mid\right.$ $\left.i=1, \cdots, 2^{n-3}\right\}$ is the set of all representatives of $G$-conjugate classes of $P$. Then, by [10, Theorems 68.4 and 65.4],

$$
k\left(B_{0}\right)=l\left(B_{0}\right)+\sum_{i=2}^{2 n-3} l\left(b_{y_{i}}\right)+\sum_{i=1}^{2 n-3} l\left(b_{z y_{i}}\right) .
$$

As in the proof of Lemma 2.2, by induction we get $l\left(b_{y_{i}}\right)=5$ for all $i=2, \cdots, 2^{n-3}$. We can assume $O(G)=1$ by Lemma 1.1. Since $Q \neq 1$, it follows from $Z^{*}$-theorem that $l\left(B_{0}\right)=5$. Since $s \in C_{G}\left(z y_{i}\right)$ and $t \notin C_{G}\left(z y_{i}\right)$, we have $e\left(C_{G}\left(z y_{i}\right)\right)=3$. Hence $l\left(b_{z y_{i}}\right)=3$ for all $i=1, \cdots, 2^{n-3}$ from Theorem 2.4(1). Thus $k\left(B_{0}\right)=2^{n}$.

LEMMA 4.3. Let $S$ be a normal subgroup of $G$ of odd index such that $S \cong J_{1} \times\left(P /\left(Z_{2} \times Z_{2} \times Z_{2}\right)\right)$. If $e=21$, then $B_{0} \cong B_{0}(S)$.

Proof. We can assume $S=J_{1} \times Q$ where $Q \cong P /\left(Z_{2} \times Z_{2} \times Z_{2}\right)$. We use induction on $|G|$. Assume $G \neq S$. By [12, Theorem], $G$ has a normal subgroup $H$ of odd prime index $l$ with $S \subseteq H$. Let $b_{0}=B_{0}(H)$. By induction, $b_{0} \cong B_{0}(S)$. Let $s$, $t, x, z$ and $y_{i}$ be the same as in the proof of Lemma 4.2. Since $z$ is an involution in $J_{1}$, by [16, Theorem], $C_{J_{1}}(z)=A_{5} \times\langle z\rangle$ where $A_{5}$ is the alternating group of degree 5. Hence $C_{S}(z)=A_{5} \times\langle z\rangle \times Q$. Let $M=C_{G}(z)$. Clearly $C_{S}(z) \cong A_{5} \times$ $\left(P /\left(Z_{2} \times Z_{2}\right)\right)$ and $C_{S}(z)$ is a normal subgroup of $M$ of odd index. By the proof of Lemma $4.2, e(M)=3$. Hence, by Lemma 2.3 , we get that $b_{z}=B_{0}(M) \cong B_{0}\left(A_{5} \times\right.$ $\left.\left(P / Z_{2} \times Z_{2}\right)\right)$ ) since $A_{5} \cong L_{2}(5)$. By Lemma 1.16(ii), the Cartan matrix of $B_{0}\left(A_{5}\right)$ has the form

$\begin{array}{rlll} & 1 & & \\ 1 & 4 & 2 & 2 \\ & 2 & 2 & 1 \\ 2 & 1 & 2 .\end{array}$

Thus, by [10, Lemma 66.1], the Cartan matrix $C_{z}$ of $b_{z}$ has the form

$$
\begin{array}{llll}
1 & 2^{n} & 2^{n-1} & 2^{n-1} \\
& 2^{n-1} & 2^{n-1} & 2^{n-2} \\
& 2^{n-1} & 2^{n-2} & 2^{n-1}
\end{array}
$$


By Lemma 4.2, $k\left(B_{0}\right)=2^{n}$. Let $\left\{\chi_{1}, \cdots, \chi_{2 n}\right\}=\operatorname{Irr}\left(B_{0}\right)$. We can write $\operatorname{IBr}\left(b_{z}\right)=$ $\left\{\phi_{1}^{z}=1_{M}, \phi_{2}^{z}, \phi_{3}^{z}\right\}$ with $\phi_{2}^{z}(1)=\phi_{3}^{z}(1)=2$ from Lemma 1.16(i). For each $\chi_{i}$ and $\phi_{\alpha}^{z}$, let $n_{i \alpha}=d_{i \alpha}^{z}$ be the generalized decomposition number of $B_{0}$ relative to $z$. Since $|z|=2$, every $n_{i \alpha}$ is an integer. Let $N=\left(n_{i \alpha}\right)_{\substack{1 \leq i \leq 2^{n} \\ 1 \leq \alpha \leq 3}}, N_{\alpha}=\left(n_{i \alpha}\right)_{1 \leq i \leq 2^{n}}$ for each $\alpha$, and $N_{\alpha} N_{\beta}=\sum_{i=1}^{2 n} n_{i \alpha} n_{i \beta}$ for each $\alpha, \beta$. It follows from [10, Theorems 63.3(2), 63.2 and 65.4] that $N_{1} N_{1}=2^{n}, N_{2} N_{2}=N_{3} N_{3}=2^{n-1}, N_{1} N_{2}=N_{1} N_{3}=2^{n-1}$ and $N_{2} N_{3}=2^{n-2}$. For each $\chi_{i}, \chi_{j}$, let $a_{i j}=\Sigma_{1 \leq \alpha, \beta \leq 3} 2^{n} n_{i \alpha} u_{\alpha \beta} n_{j \beta}$, where $C_{z}^{-1}=\left(u_{\alpha \beta}\right)_{1 \leq \alpha, \beta \leq 3}$. Then

$$
\begin{aligned}
a_{i i} & =3 n_{i 1}{ }^{2}+4\left(n_{i 2}{ }^{2}+n_{i 3}{ }^{2}\right)-4\left(n_{i 1} n_{i 2}+n_{i 1} n_{i 3}\right) \\
& \equiv n_{i 1}{ }^{2} \equiv n_{i 1}(\bmod 2)
\end{aligned}
$$

for all $\chi_{i}$. By Lemma 1.11, every $\chi_{i}$ has height zero. Hence, by [4, II (7A) and $(5 \mathrm{G})]$, every $a_{i i}$ is odd, so that $n_{i 1}$ is odd for all $i=1, \cdots, 2^{n}$. Since $N_{1} N_{1}=2^{n}$, $n_{i 1}= \pm 1$ for all $i=1, \cdots, 2^{n}$. Let $\delta_{i}=n_{i 1}$ and $u_{i}=n_{i 2} \delta_{i}$ for each $i$. Since $N_{1} N_{2}=$ $N_{2} N_{2}=2^{n-1}, \sum_{i=1}^{2 n} u_{i}=\sum_{i=1}^{2 n} u_{i}{ }^{2}$. Thus, $u_{i}=1$ or 0 for all $i=1, \cdots, 2^{n}$. Hence exactly $2^{n-1} u_{i}$ 's are 1 and the other $u_{i}$ 's are 0 since $N_{1} N_{2}=2^{n-1}$. Then we may assume

$$
n_{i 2}= \begin{cases}\delta_{i} & \text { for } i=1, \cdots, 2^{n-1} \\ 0 & \text { for } i=2^{n-1}+1, \cdots, 2^{n} .\end{cases}
$$

Similarly, exactly $2^{n-1}\left(n_{i 3} \delta_{i}\right)$ 's are 1 and the other $\left(n_{i 3} \delta_{i}\right)$ 's are 0 . Since $N_{2} N_{3}$ $=2^{n-2}$, we may assume

$$
n_{i 3}=\left\{\begin{array}{l}
\delta_{i} \text { for } i=1, \cdots, 2^{n-2} \text { and for } i=2^{n-1}+1, \cdots, 3 \cdot 2^{n-2} \\
0 \text { for } i=2^{n-2}+1, \cdots, 2^{n-1} \text { and for } i=3 \cdot 2^{n-2}+1, \cdots, 2^{n} .
\end{array}\right.
$$

Since $\chi_{i}(z)=n_{i 1}+2\left(n_{i 2}+n_{i 3}\right)$ for each $i$, we get

$$
\chi_{i}(z)= \begin{cases} \pm 5 & \text { for } i=1, \cdots, 2^{n-2} \\ \pm 3 & \text { for } i=2^{n-2}+1, \cdots, 3 \cdot 2^{n-2} \\ \pm 1 & \text { for } i=3 \cdot 2^{n-2}+1, \cdots, 2^{n}\end{cases}
$$

Let $C_{G}(P)=P \times V$. When $G=V H, G=C_{G}(P) \cdot H$, so that $B_{0} \cong b_{0}$ from Proposition 1.6. Thus, we may assume $G \neq V H$. Hence $C_{H}(P)=P \times V$. Since $b_{0} \cong B_{0}(S)$, it follows from Proposition 1.5 that $\left|H: V H^{\prime}\right|=k^{\prime}\left(b_{0}\right)=2^{n-3}$. By [10, Theorem 18.4], $P \cap G^{\prime}=\left\{1, x, x^{t}, \cdots, x^{t^{6}}\right\}$. Then the order of Sylow 2-subgroups of $G^{\prime}$ is 8 . This implies $2^{n-3}|| G: V G^{\prime} \mid$ and $2^{n-2} \chi\left|G: V G^{\prime}\right|$. Thus, by Proposition 1.5, $k^{\prime}\left(B_{0}\right)=\left|G: V G^{\prime}\right|=l \cdot 2^{n-3}$ where $l=|G: H|$. Since $b_{0} \cong B_{0}(S)$, by Clifford's theorem, Proposition 1.3 and the character table of $J_{1}[16$, p. 148], we get that $\chi_{i}(z)=1$ for every $\chi_{i} \in \operatorname{Irr}\left(B_{0}\right)$ with degree one. These show that the number of 
$\chi_{i} \in \operatorname{Irr}\left(B_{0}\right)$ with $\chi_{i}(z)=1$ is at least $l \cdot 2^{n-3}$. However, $\chi_{i}(z)= \pm 1$ only for $i=$ $3 \cdot 2^{n-2}+1, \cdots, 2^{n}$. This is a contradiction since $l \cdot 2^{n-3}>2^{n-2}$. This completes the proof.

LEMMA 4.4. Let $S$ be a normal subgroup of $G$ of odd index such that $S \cong R(q) \times\left(P /\left(Z_{2} \times Z_{2} \times Z_{2}\right)\right)$. If $e=21$, then $B_{0} \cong B_{0}(S)$.

Proof. Let $R=R(q)$. We may assume $S=R \times Q$ where $Q \cong P /\left(Z_{2} \times Z_{2} \times Z_{2}\right)$. We prove the lemma by induction on $|G|$. Assume $G \neq S$. By [12, Theorem], $G$ has a normal subgroup $H$ of odd prime index $l$ with $S \cong H$. Let $b_{0}=B_{0}(H)$. By induction, $b_{0} \cong B_{0}(S)$. Let $s, t, x, z$ and $y_{i}$ be the same as in the proof of Lemma 4.2. Since $z$ is an involution in $R, C_{R}(z)=L_{2}(q) \times\langle z\rangle$ from $[21$, p. 62 III]. (It is noted that we use the notation $R(q)$ as in the sense of [13]). Hence $C_{S}(z)=L_{2}(q) \times\langle z\rangle \times Q$. Let $M=C_{G}(z)$. Then $C_{S}(z)$ is a normal subgroup of $M$ of odd index and $C_{S}(z) \cong L_{2}(q) \times\left(P /\left(Z_{2} \times Z_{2}\right)\right)$. By the proof of Lemma 4.2, $e(M)=3$. Then, by Lemma 2.3, $b_{z}=B_{0}(M) \cong B_{0}\left(L_{2}(q) \times\left(P /\left(Z_{2} \times Z_{2}\right)\right)\right)$. By [21, Theorem (1)], $3<q \equiv 3(\bmod 8)$, so that as in the proof of Lemma 4.3 the Cartan matrix $C_{z}$ of $b_{z}$ has the form

$$
\begin{array}{lll}
2^{n-1} & 2^{n-2} & 2^{n-2} \\
2^{n-2} & 2^{n-1} & 2^{n-2} \\
2^{n-2} & 2^{n-2} & 2^{n-1}
\end{array}
$$

By Lemma 4.2, $k\left(B_{0}\right)=2^{n}$. Let $\left\{\chi_{1}, \cdots, \chi_{2 n}\right\}=\operatorname{Irr}\left(B_{0}\right)$. We can write $\operatorname{IBr}\left(b_{z}\right)=$ $\left\{\phi_{1}^{z}=1_{M}, \phi_{2}^{z}, \phi_{3}^{z}\right\}$ with $\phi_{2}^{z}(1)=\phi_{3}^{z}(1)=(q-1) / 2$ from Lemma 1.16(i). Let $n_{i \alpha}, N, N_{\alpha}$ and $N_{\alpha} N_{\beta}$ be the same as in the proof of Lemma 4.3. Every $n_{i \alpha}$ is an integer. As in the proof of Lemma 4.3 we get $N_{\alpha} N_{\alpha}=2^{n-1}$ for all $\alpha=1,2,3$, and $N_{\alpha} N_{\beta}$ $=2^{n-2}$ if $\alpha \neq \beta$. Let $C_{G}(P)=P \times V$. As in the proof of Lemma 4.3 we may assume $G \neq V H$. Since $b_{0} \cong B_{0}(S), k^{\prime}\left(b_{0}\right)=2^{n-3}$. So that $k^{\prime}\left(B_{0}\right)=\left|G: V G^{\prime}\right|=l \cdot 2^{n-3}$ as in the proof of Lemma 4.3, where $l=|G: H|$. Since $b_{0} \cong B_{0}(S)$, by $[21$, p. 74 and pp. 87-88], we can write $\left\{\tilde{\chi}_{i j} \mid i=1, \cdots, 8 ; j=1, \cdots, 2^{n-3}\right\}=\operatorname{Irr}\left(b_{0}\right)$ and

$\begin{array}{lcc} & 1 & z \\ \tilde{\chi}_{1 j} & 1 & 1 \\ \tilde{\chi}_{2 j} & q^{2}-q+1 & -1 \\ \tilde{\chi}_{3 j} & q^{3} & q \\ \tilde{\chi}_{4 j} & q\left(q^{2}-q+1\right) & -q \\ \tilde{\chi}_{5 j} & (q-1) m(q+1+3 m) / 2 & -(q-1) / 2 \\ \tilde{\chi}_{6 j} & (q-1) m(q+1+3 m) / 2 & -(q-1) / 2 \\ \tilde{\chi}_{7 j} & (q-1) m(q+1-3 m) / 2 & (q-1) / 2 \\ \tilde{\chi}_{8 j} & (q-1) m(q+1-3 m) / 2 & (q-1) / 2\end{array}$


for $j=1, \cdots, 2^{n-3}$, where $q=3^{2 k+1}$ and $m=3^{k}$ for some $k \geqq 1$ (cf. [21, Theorem]). By Clifford's theorem, Proposition 1.3 and the above table, we know that if $\chi_{i}(1)=1$ then $\chi_{i}(z)=1$. When $n_{i_{1}}=0, \chi_{i}(z)=\left(n_{i 2}+n_{i 3}\right)(q-1) / 2$. Thus $n_{i_{1}} \neq 0$ if $\chi_{i}(z)= \pm 1$. Hence the number of $\chi_{i} \in \operatorname{Irr}\left(B_{0}\right)$ with $n_{i 1} \neq 0$ is at least $l \cdot 2^{n-3}$. Since $N_{1} N_{1}=2^{n-1}$, we get $l=3$. Fix any $\chi_{i}$. If $\left.\chi_{i}\right|_{H}=\tilde{\chi}_{2 j}$ for some $j$ with $1 \leqq j \leqq 2^{n-3}$, then $n_{i 1}{ }^{2} \geqq 1$ since $\chi_{i}(z)=-1$. If $\left.\chi_{i}\right|_{H}=\tilde{\chi}_{2 j}+\tilde{\chi}_{2 j^{\prime}}+\tilde{\chi}_{2 j^{\prime}}$ for some $j, j^{\prime}, j^{\prime \prime}$ with $1 \leqq j<j^{\prime}<j^{\prime \prime} \leqq 2^{n-3}$, then $n_{i 1}{ }^{2} \geqq 9$ since $\chi_{i}(z)=-3$. Let $u$ be the number of $\chi_{i} \in \operatorname{Irr}\left(B_{0}\right)$ with $\left.\chi_{i}\right|_{H}=\tilde{\chi}_{2 j}$, and let $v$ be the number of $\chi_{i} \in \operatorname{Irr}\left(B_{0}\right)$ with $\left.\chi_{i}\right|_{H}=$ $\tilde{\chi}_{2 j}+\tilde{\chi}_{2 j^{\prime}}+\tilde{\chi}_{2 j^{\prime \prime}}$ for $j<j^{\prime}<j^{\prime \prime}$. Since $N_{1} N_{1}=2^{n-1}$, and since $1<q^{2}-q+1<3\left(q^{2}-q+1\right)$, we have

$$
2^{n-1}=\sum_{i=1}^{2 n} n_{i 1}{ }^{2} \geqq k^{\prime}\left(B_{0}\right)+u+9 v=3 \cdot 2^{n-3}+u+9 v .
$$

Then $2^{n-3} \geqq u+9 v$. By Proposition 1.4, for every $\tilde{\chi}_{2 j}$ there is some $\chi_{i}$ with $\left(\left.\chi_{i}\right|_{H I}, \tilde{\chi}_{2 j}\right) \neq 0$, so that, by Clifford's theorem and Proposition $1.3,\left.\chi_{i}\right|_{H}=\tilde{\chi}_{2 j}$ or $\left.\chi_{i}\right|_{I I}=\tilde{\chi}_{2 j}+\tilde{\chi}_{2 j}{ }^{g}+\tilde{\chi}_{2 j}{ }^{2}$ where $g$ is an element of $G$ with $G=\langle g, H\rangle$. By considering the degrees of $\tilde{\chi}_{i j}$, we get that $\tilde{\chi}_{2 j^{g}}$ and $\tilde{\chi}_{2 j}{ }^{2}$ are both in $\left\{\tilde{\chi}_{2 j^{\prime}} \mid j^{\prime}=1, \cdots, 2^{n-3}\right\}$. Thus $2^{n-3} \leqq u+3 v$, so that $v=0$ and $u=2^{n-3}$. This implies that the number of $\chi_{i} \in \operatorname{Irr}\left(B_{0}\right)$ with $\chi_{i}(z)=-1$ is at least $2^{n-3}$, so that the number of $\chi_{i} \in \operatorname{Irr}\left(B_{0}\right)$ with $\chi_{i}(z)= \pm 1$ is at least $2^{n-1}$. Then the number of $\chi_{i} \in \operatorname{Irr}\left(B_{0}\right)$ with $n_{i 1} \neq 0$ is at least $2^{n-1}$. Since $N_{1} N_{1}=2^{n-1}$, we may assume

$$
n_{i 1}=\left\{\begin{array}{l}
\delta_{i} \text { for } i=1, \cdots, 2^{n-1} \\
0 \text { for } i=2^{n-1}+1, \cdots, 2^{n}
\end{array}\right.
$$

where $\delta_{i}= \pm 1$. Thus $\chi_{i}(z)= \pm 1$ for all $i=1, \cdots, 2^{n-1}$. For all $i=1, \cdots, 2^{n-1}$, $\chi_{i}(z)=\delta_{i}+\left(n_{i 2}+n_{i 3}\right)(q-1) / 2$, so that $n_{i 2}+n_{i 3}=0$ since $(q-1) / 2 \geqq 13$. Consequently, $N_{1} N_{2}+N_{1} N_{3}=\sum_{i=1}^{2 n} n_{i 1}\left(n_{i 2}+n_{i 3}\right)=\sum_{i=1}^{2 n-1} \delta_{i}\left(n_{i 2}+n_{i 3}\right)=0$. This is a contradiction since $N_{1} N_{2}=N_{1} N_{3}=2^{n-2}$. This completes the proof.

LEMMA 4.5. Let $S$ be a normal subgroup of $G$ of odd index such that $S \cong L_{2}(q) \times S L(2,8)$ for some $q>3$ with $q \equiv 3$ or $5(\bmod 8)$. If $e=21$, then $B_{0} \cong B_{0}(S)$.

Proof. Let $R_{1}$ and $R_{2}$ be Sylow 2-subgroups of $L_{2}(q)$ and $S L(2,8)$, respectively. We may assume $S=L_{2}(q) \times S L(2,8)$ and $P=R_{1} \times R_{2}$. There are an element $s \in L_{2}(q)$ and an involution $x \in R_{1}$ with $R_{1}=\left\{1, x, x^{s}, x^{s^{2}}\right\}$. Similarly, we can write $R_{2}=\left\{1, y, y^{t}, \cdots, y^{t^{6}}\right\}$ for some $t \in S L(2,8)$ and for an involution $y \in R_{2}$. Since $e=21, N_{G}(P)=\left\langle s, t, C_{G}(P)\right\rangle$ and $N_{G}(P) / C_{G}(P)$ is cyclic of order 21 . By [10, Lemma 18.5], $\{1, x, y, x y\}$ is the set of all representatives of $G$-conjugate classes of $P$. Hence, by [10, Theorems 68.4 and 65.4$], k\left(B_{0}\right)=l\left(B_{0}\right)+l\left(b_{x}\right)$ 
$+l\left(b_{y}\right)+l\left(b_{x y}\right)$. Since $s \notin C_{G}(x)$ and $t \in C_{G}(x)$, we have $e\left(C_{G}(x)\right)=7$. Thus $l\left(b_{x}\right)=7$ from Theorem 2.4(1). Similarly, $l\left(b_{y}\right)=3$ from Theorem 2.4(1). For integers $i$ and $j,(x y)^{s^{i} t^{j}}=x y$ if and only if $3 \mid i$ and $7 \mid j$. This implies $N_{M}(P)=C_{M}(P)$ where $M=C_{G}(x y)$. Thus, by [10, Theorem 18.7 and Corollary 65.3], $l\left(b_{x y}\right)=l\left(B_{0}(M)\right)=1$. Since $G$ is nonsolvable, $l\left(B_{0}\right) \geqq 2$ from $[10$, Corollary 65.3$]$, so that $k\left(B_{0}\right) \geqq 13$.

Now, we prove the lemma by induction on $|G|$. Assume $G \neq S$. By $[12$, Theorem], $G$ has a normal subgroup $H$ of odd prime index $l$ with $S \subseteq H$. Let $b_{0}=B_{0}(H)$. We know $b_{0} \cong B_{0}(S)$ by induction. From the character tables of $L_{2}(q)$ and $S L(2,8)$ (cf. [10, Theorems 38.1 and 38.2]), we can write

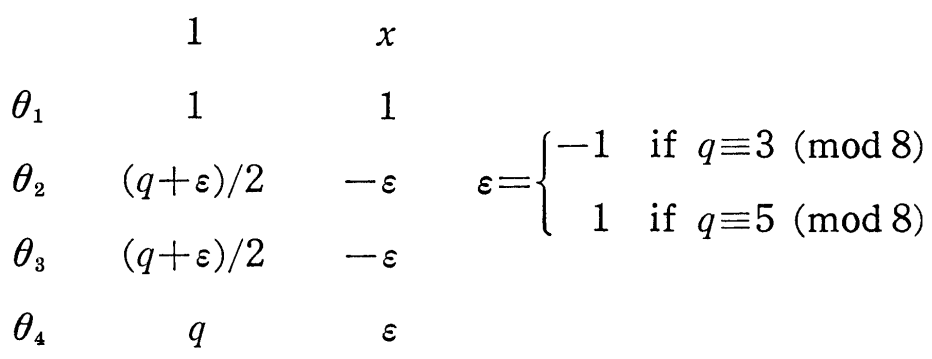

where $\left\{\theta_{1}, \theta_{2}, \theta_{3}, \theta_{4}\right\}=\operatorname{Irr}\left(B_{0}\left(L_{2}(q)\right)\right)$ and

$$
\begin{array}{rrrl} 
& 1 & y & \\
\zeta_{1} & 1 & 1 & \\
\zeta_{j} & 7 & -1 & \text { for } j=2,3,4,5 \\
\zeta_{j} & 9 & 1 & \text { for } j=6,7,8
\end{array}
$$

where $\left\{\zeta_{1}, \cdots, \zeta_{8}\right\}=\operatorname{Irr}\left(B_{0}(S L(2,8))\right)$. Since $b_{0} \cong B_{0}(S)$, we may write $\operatorname{Irr}\left(b_{0}\right)=$ $\left\{\tilde{\chi}_{i j} \mid i=1, \cdots, 4 ; j=1, \cdots, 8\right\}$ with $\left.\tilde{\chi}_{i j}\right|_{S}=\theta_{i} \zeta_{j}$ for all $i, j$. Hence the degrees of all $\tilde{\chi}_{i j}$ are $1,7,9,(q+\varepsilon) / 2,7(q+\varepsilon) / 2,9(q+\varepsilon) / 2, q, 7 q$ and $9 q$. Next, we want to show that

$$
\text { if } \tilde{\chi}, \tilde{\chi}^{\prime} \in \operatorname{Irr}\left(b_{0}\right) \text { with } \tilde{\chi}(1)=\tilde{\chi}^{\prime}(1)
$$

then $\tilde{\chi}(x y)=\tilde{\chi}^{\prime}(x y)= \pm 1$.

Case 1. $\varepsilon=1$ : Clearly $\{1,9,7(q+1) / 2,9,9 q\} \cap\{7,(q+1) / 2,9(q+1) / 2,7 q\}=\varnothing$. Hence, by considering the values $\tilde{\chi}_{i j}(1)$ and $\tilde{\chi}_{i j}(x y)$, we get $\left(^{*}\right)$.

Case 2. $\varepsilon=-1$ : Since $\{1,9,(q-1) / 2,9(q-1) / 2,7 q\} \cap\{7,7(q-1) / 2, q, 9 q\}=\varnothing$, we obtain $\left(^{*}\right)$ as in Case 1.

We get from Clifford's theorem, Proposition 1.3, $\left(^{*}\right)$ and the above character tables of $L_{2}(q)$ and $S L(2,8)$ that $\chi(x y)= \pm 1$ or $\pm l$ for every $\chi \in \operatorname{Irr}\left(B_{0}\right)$. Let $k=k\left(B_{0}\right)$, and let $m$ be the number of $\chi \in \operatorname{Irr}\left(B_{0}\right)$ with $\chi(x y)= \pm 1$. Hence we can write $\operatorname{Irr}\left(B_{0}\right)=\left\{\chi_{1}=1_{G}, \chi_{2}, \cdots, \chi_{m}, \chi_{m+1}, \cdots, \chi_{k}\right\}$ such that 


$$
\chi_{i}(x y)= \begin{cases} \pm 1 & \text { for } i=1, \cdots, m \\ \pm l & \text { for } i=m+1, \cdots, k\end{cases}
$$

Since $l\left(b_{x y}\right)=1$, as in the proof of Lemma 1.9,

$$
32=\sum_{i=1}^{k} \chi_{i}(x y)^{2}=m+(k-m) l^{2} .
$$

Firstly, suppose $k=m$. Then $\chi_{i}(x y)= \pm 1$ for all $\chi_{i} \in \operatorname{Irr}\left(B_{0}\right)$. Since $k=m=32$ and since $b_{0} \cong B_{0}(S)$, we have $k\left(B_{0}\right)=k\left(b_{0}\right)=32$. Hence $l\left(B_{0}\right)=21$, so that $l\left(B_{0}\right)=$ $l\left(b_{0}\right)$ since $b_{0} \cong B_{0}(S)$. Thus, by $(*)$ and Corollary $1.8, B_{0} \cong b_{0}$. Thus, we may assume $k>m$. Since $k \geqq 13$, by $\left.{ }^{* *}\right), l=3$. So that $k-m=1$ or 2 . Let $C_{G}(P)=$ $P \times V$. Since $k>m$ and $b_{0} \cong B_{0}(S)$, we know $B_{0} \cong b_{0}$. Hence $G \neq V H$ from Proposition 1.6. This shows $C_{H}(P)=P \times V$. Thus, by Proposition 1.5, $\left|H: V H^{\prime}\right|=$ $k^{\prime}\left(b_{0}\right)=1$ since $b_{0} \cong B_{0}(S)$. Then $H=V H^{\prime}$. Since $G / H$ is cyclic, $V G^{\prime}=V H^{\prime}=H$. Hence $k^{\prime}\left(B_{0}\right)=\left|G: V G^{\prime}\right|=l=3$ by Proposition 1.5. So that we may assume that $\chi_{1}(1)=\chi_{2}(1)=\chi_{3}(1)=1$ and $\chi_{i}(1)>1$ for all $i=4, \cdots, k$.

Case A. $k-m=1$ : By (**), we get $m=23$ and $k=24$. Then $\chi_{i}(x y)= \pm 1$ for $i=1, \cdots, 23$ and $\chi_{24}(x y)= \pm 3$. Since $b_{0} \cong B_{0}(S)$, by Clifford's theorem and Proposition 1.3, $\left.\chi_{i}\right|_{H}=1_{H}$ for $i=1,2,3,\left.\chi_{i}\right|_{H} \neq 1_{H}$ and $\left.\chi_{i}\right|_{H} \in \operatorname{Irr}\left(b_{0}\right)$ for $i=4, \cdots, 23$, $\left.\chi_{24}\right|_{H}=\tilde{\chi}_{i_{1}}+\tilde{\chi}_{i_{2}}+\tilde{\chi}_{i_{3}}$ where $\tilde{\chi}_{i_{1}}, \tilde{\chi}_{i_{2}}$ and $\tilde{\chi}_{i_{3}}$ are distinct $G$-conjugate elements in $\operatorname{Irr}\left(b_{0}\right)$. On the other hand, it follows from Proposition 1.4 that for every $\tilde{\chi} \in \operatorname{Irr}\left(b_{0}\right)$ there is some $\chi_{i} \in \operatorname{Irr}\left(B_{0}\right)$ with $\left(\left.\chi_{i}\right|_{H}, \tilde{\chi}\right) \neq 0$. These show $k\left(b_{0}\right) \leqq 1+20+3$ $=24$. But $k\left(b_{0}\right)=32$ since $b_{0} \cong B_{0}(S)$. Then we have a contradiction.

Case B. $k-m=2$ : We have from $(* *)$ that $\chi_{i}(x y)= \pm 1$ for $i=1, \cdots, 14$, $\chi_{15}(x y)= \pm 3$ and $\chi_{16}(x y)= \pm 3$. Hence as in Case A we get $k\left(b_{0}\right) \leqq 1+11+6=18$. This is a contradiction as in Case A. This completes the proof.

LEMMA 4.6. Let $S$ be a normal subgroup of $G$ of odd index such that $S \cong L_{2}(q) \times S L(2,8) \times\left(P /\left(Z_{2} \times Z_{2} \times Z_{2} \times Z_{2} \times Z_{2}\right)\right)$ for some $q>3$ with $q \equiv 3$ or 5 (mod 8). If $e=21$, then $k\left(B_{0}\right)=2^{n}$ and $l\left(B_{0}\right)=21$.

Proof. If $n=5$, we can prove the lemma by Lemma 4.5 (cf. Lemma 1.12 and Theorem 2.4). If $n>5$, we can verify the lemma by induction on $n$ as in the proof of Lemma 4.2 .

LemmA 4.7. Assume as in Lemma 4.6. Then $B_{0} \cong B_{0}(S)$.

Proof. We may assume $S=L_{2}(q) \times S L(2,8) \times Q$ with $Q \cong P /\left(Z_{2} \times Z_{2} \times Z_{2} \times Z_{2}\right.$ $\times Z_{2}$ ). We use induction on $|G|$ as before. Assume $G \neq S$. Hence $G$ has a normal subgroup $H$ of odd prime index with $S \subseteq H$ from [12, Theorem]. Let $b_{0}=B_{0}(H)$. By induction, $b_{0} \cong B_{0}(S)$. Let $x$ and $y$ be involutions in $L_{2}(q)$ and 
$S L(2,8)$, respectively. As in the proof of Lemma 4.2, $l\left(b_{x y}\right)=1$. By Lemma 4.6, $k\left(B_{0}\right)=2^{n}$. Thus, $\chi(x y)= \pm 1$ for all $\chi \in \operatorname{Irr}\left(B_{0}\right)$ from Lemma 1.9. By Lemma 4.6, $k\left(B_{0}\right)=k\left(b_{0}\right)$ and $l\left(B_{0}\right)=l\left(b_{0}\right)$. Since $b_{0} \cong B_{0}(S)$, as in the proof of Lemma 4.5 , we get that if $\tilde{\chi}, \tilde{\chi}^{\prime} \in \operatorname{Irr}\left(b_{0}\right)$ with $\tilde{\chi}(1)=\tilde{\chi}^{\prime}(1)$ then $\tilde{\chi}(x y)=\tilde{\chi}^{\prime}(x y)= \pm 1$. These imply $B_{0} \cong b_{0}$ from Corollary 1.8. This completes the proof.

Next, we state the following main result of this section. That is proved by making use of Lemmas $4.2-4.7$.

THEOREM 4.8. Let $P$ be an abelian Sylow 2-subgroup of $G$, and let $S=$ $O^{\prime}(G / O(G))$. If $e=e(S)=21$, then we have the following.

(1) $k\left(B_{0}\right)=|P|$ and

$$
l\left(B_{0}\right)=\left\{\begin{aligned}
5 & \text { if } N_{G}(P) / C_{G}(P) \text { is noncyclic } \\
21 & \text { if } N_{G}(P) / C_{G}(P) \text { is cyclic. }
\end{aligned}\right.
$$

(2) One of the following holds:

(i ) $B_{0} \cong B_{0}\left(J_{1} \times\left(P /\left(Z_{2} \times Z_{2} \times Z_{2}\right)\right)\right)$,

(ii) $B_{0} \cong B_{0}\left(R(q) \times\left(P /\left(Z_{2} \times Z_{2} \times Z_{2}\right)\right)\right)$,

(iii) $B_{0} \cong B_{0}\left(L_{2}(q) \times S L(2,8) \times\left(P /\left(Z_{2} \times Z_{2} \times Z_{2} \times Z_{2} \times Z_{2}\right)\right)\right)$ for some $q>3$ with $q \equiv 3$ or $5(\bmod 8)$.

Proof. By Lemma 1.1, we may assume $O(G)=1$. By Proposition 1.10 and Lemma 1.12, one of the following holds:

(i ) $S \cong J_{1} \times\left(P /\left(Z_{2} \times Z_{2} \times Z_{2}\right)\right)$,

(ii) $S \cong R(q) \times\left(P /\left(Z_{2} \times Z_{2} \times Z_{2}\right)\right)$,

(iii) $S \cong L_{2}(q) \times S L(2,8) \times\left(P /\left(Z_{2} \times Z_{2} \times Z_{2} \times Z_{2} \times Z_{2}\right)\right)$ for some $q>3$ with $q \equiv 3$ or $5(\bmod 8)$. Then we can prove the theorem by Lemmas 4.2-4.7.

\section{The case when $P$ is elementary abelian of order 8}

In this section we consider the case when $G$ has elementary abelian Sylow 2-subgroups of order 8 . In particular, we shall determine $B_{0}$ in the case when $G$ is nonsolvable, $e=21$ and $e(S) \neq 21$ where $S=O^{\prime}(G / O(G))$. Throughout this section we assume that $G$ has an elementary abelian Sylow 2-subgroup $P$ of order 8 and we use the notation $e$ and $B_{0}$ as before.

By Lemma 1.14 and Remark 1 of $\S 1$, it is sufficient to consider the cases when $e=3,7$ and 21 .

Proposition 5.1. (i ) If $e=3$, then $k\left(B_{0}\right)=8$ and $l\left(B_{0}\right)=3$.

(ii) If $e=7$, then $k\left(B_{0}\right)=8$ and $l\left(B_{0}\right)=7$. 
(iii) If $e=21$, then $k\left(B_{0}\right)=8$ and $l\left(B_{0}\right)=5$.

Proof. (i ) We can write $N_{G}(P)=\left\langle s, C_{G}(P)\right\rangle$ for some $s \in N_{G}(P)$. There is an involution $x \in P$ with $x^{s} \neq x$. Hence $l\left(b_{x}\right)=1$ as in the proof of Lemma 2.1. Then $k\left(B_{0}\right)=8$ from Lemma $1.15(1)$. On the other hand, $l\left(B_{0}\right)=3$ by Theorem 2.4(1).

(ii) We can verify (ii) as in (i).

(iii) We have already proved (iii) in Lemma 4.1.

Proposition 5.2. There is a basic set $W$ of $B_{0}$ such that $W$ contains the trivial Brauer character and the decomposition matrix of $B_{0}$ with respect to $W$ has the form

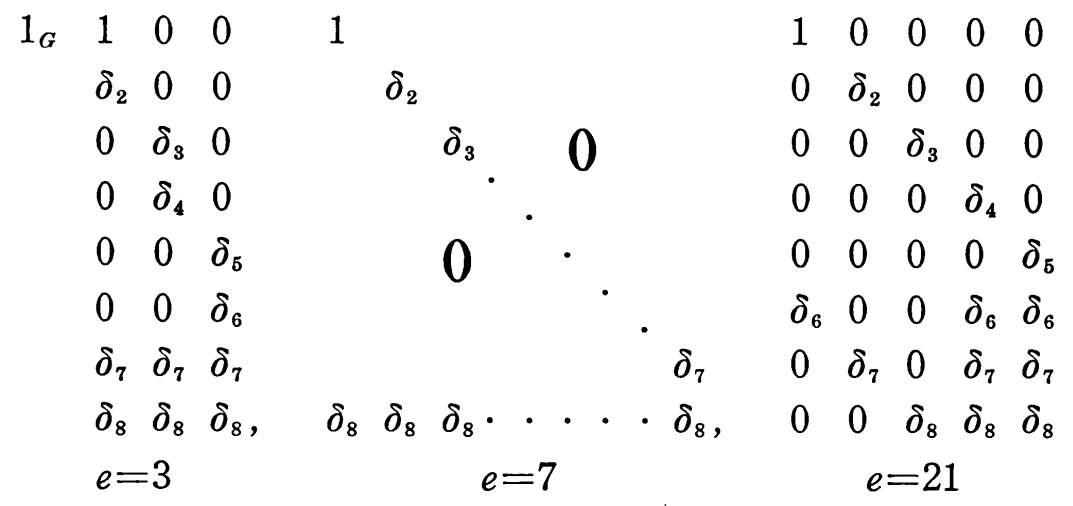

where $\delta_{i}= \pm 1$.

Proof. Case 1. $e=3$ : Clear from Proposition 5.1(i) and the proof of Lemma 4.1.

Case 2. $e=7$ : By Proposition 5.1(ii), $k\left(B_{0}\right)=8$. Let $\left\{\chi_{1}, \cdots, \chi_{8}\right\}=\operatorname{Irr}\left(B_{0}\right)$. By the proof of Proposition 5.1(ii), $G$ has an involution $x$ with $l\left(b_{x}\right)=1$. By Lemma 1.9 , we get $\chi_{i}(x)= \pm 1$ for all $i$. On the other hand, $\sum_{i=1}^{8} \chi_{i}(x) \chi_{i}=0$ on $2^{\prime}$-elements of $G$ from [10, Theorem 63.3(1)]. Thus, the assertion is proved.

Case. $3 e=21$ : Let $z$ be an involution in $G$. By the proof of Lemma 4.1, the generalized decomposition matrix of $B_{0}$ relative to $z$ with respect to some basic set of $b_{z}$ has the same form as in Case 1. Hence, by [10, Theorem 63.3(1)], we can verify the proposition.

LEMmA 5.3. Assume $e=21, O(G)=1, O^{\prime}(G)=S L(2,8)$ and $G$ has a normal subgroup $H$ of odd prime index with $e(H)=7$. Then for any involution $z$ in $G$ we get 


$$
\chi_{i}(1)=\left\{\begin{array}{rl}
1 & \text { for } i=1,2,3 \\
7 & \text { for } i=4,5,6 \\
21 & \text { for } i=7 \\
27 & \text { for } i=8,
\end{array} \quad \chi_{i}(z)=\left\{\begin{aligned}
1 & \text { for } i=1,2,3 \\
-1 & \text { for } i=4,5,6 \\
-3 & \text { for } i=7 \\
3 & \text { for } i=8
\end{aligned}\right.\right.
$$

where $\left\{\chi_{1}=1_{G}, \chi_{2}, \cdots, \chi_{8}\right\}=\operatorname{Irr}\left(B_{0}\right)$.

Proof. Let $S=O^{\prime}(G)=S L(2,8)$. By Lemmas 1.12 and 1.14 , we can write $N_{S}(P)=\left\langle s, C_{S}(P)\right\rangle, N_{H}(P)=\left\langle s, C_{H}(P)\right\rangle$ and $N_{G}(P)=\left\langle s, t, C_{G}(P)\right\rangle$ for some $s \in N_{S}(P)$ and $t \in N_{G}(P)$ such that $s$ and $t$ have orders 7 and 3 modulo $C_{G}(P)$, respectively. Clearly, $G / H=\langle t H\rangle$. Let $b_{0}=B_{0}(H)$, and let $C_{G}(P)=P \times V$. By Proposition 5.2, $B_{0} \cong b_{0}$. Hence $V H=H$ from Proposition 1.6. Then $C_{G}(P)=C_{H}(P)$ and $|G: H|=3$. We may assume $z \in P$. Let $M=C_{G}(z)$. By the proof of Lemma 2.1, $C_{S}(z)$ is a 2-nilpotent normal subgroup of $M$, so that $M$ is solvable. By the proof of Lemma $4.1, e(M)=3$. Thus, by Lemma $1.1, B_{0}(M) \cong B_{0}\left(P \cdot Z_{3}\right)$ where $P \cdot Z_{3}$ is the semi-direct product of its normal subgroup $P$ by $Z_{3}$ and it is not the direct product $P \times Z_{3}$. Thus, as in the proof of Lemma 4.1 we know the generalized decomposition numbers of $B_{0}$ relative to $z$. So we can write

$$
\chi_{i}(z)= \begin{cases} \pm 1 & \text { for } i=1, \cdots, 6 \\ \pm 3 & \text { for } i=7,8\end{cases}
$$

for suitable indexing of $\chi_{2}, \cdots, \chi_{8}$. By Lemma 2.1, $b_{0} \cong B_{0}(S)$. Hence, by $[10$, Theorem 38.2],

$$
\tilde{\chi}_{i}(1)=\left\{\begin{array}{ll}
1 & \text { for } i=1 \\
7 & \text { for } i=2, \cdots, 5 \\
9 & \text { for } i=6,7,8
\end{array} \quad \tilde{\chi}_{i}(z)=\left\{\begin{aligned}
1 & \text { for } i=1 \\
-1 & \text { for } i=2, \cdots, 5 \\
1 & \text { for } i=6,7,8
\end{aligned}\right.\right.
$$

where $\left\{\tilde{\chi}_{1}, \cdots, \tilde{\chi}_{8}\right\}=\operatorname{Irr}\left(b_{0}\right)$. Since $|G: V H|=|G: H|=3$, we get 3||$G: V G^{\prime} \mid$. By Proposition 1.5, $k^{\prime}\left(B_{0}\right)=\left|G: V G^{\prime}\right|$. By $\left.{ }^{* *}\right), k^{\prime}\left(b_{0}\right)=1$, so that $\left|G: V G^{\prime}\right|=3$ from Frobenius reciprocity. So we may assume that $\left.\chi_{1}\right|_{H}=\left.\chi_{2}\right|_{H}=\left.\chi_{3}\right|_{H}=\tilde{\chi}_{1}$ from (*), (**) and Proposition 1.3. Similarly, we may also assume that $\left.\chi_{7}\right|_{H}=\tilde{\chi}_{3}+\tilde{\chi}_{4}+\tilde{\chi}_{5}$ and $\left.\chi_{8}\right|_{H}=\tilde{\chi}_{6}+\tilde{\chi}_{7}+\tilde{\chi}_{8}$. Then we get $\left.\chi_{4}\right|_{H}=\left.\chi_{5}\right|_{H}=\left.\chi_{6}\right|_{H}=\tilde{\chi}_{2}$. This completes the proof.

The next theorem is the main result of this section.

THEOREM 5.4. Let $\bar{G}=G / O(G)$ and $S=O^{\prime}(\bar{G})$. If $G$ is nonsolvable, $e=21$ and $e(S) \neq 21$, then we have the following.

(i) $S \cong S L(2,8)$.

(ii) For any subnormal subgroup $\bar{L}$ of $\bar{G}$ of odd index with $e(\bar{L})=21$, 
$B_{0} \cong B_{0}(\bar{L})$.

Proof. We may assume $O(G)=1$ by Lemma 1.1 , so that $S=O^{\prime}(G)$.

(i) Noncyclic groups of order 21 have no normal subgroups of order 3. Thus, by Lemma $1.14, e(S)=7$. Then $S \cong S L(2,8)$ from Proposition 1.10 and Lemma 1.12.

(ii) Firstly, we want to show that

if $L$ is a normal subgroup of $G$ such that $|G: L|$ is an odd prime and $\{(L)=21$ and if $H$ is a normal subgroup of $L$ such that $|L: H|$ is an odd prime and $e(H)=7$, then $B_{0} \cong B_{0}(L)$.

Let $b_{0}=B_{0}(L)$, and let $z$ be an involution in $G$. By Lemma 5.3, we get

$$
\tilde{\chi}_{i}(1)=\left\{\begin{array}{rl}
1 & \text { for } i=1,2,3 \\
7 & \text { for } i=4,5,6 \\
21 & \text { for } i=7 \\
27 & \text { for } i=8,
\end{array} \quad \tilde{\chi}_{i}(z)=\left\{\begin{aligned}
1 & \text { for } i=1,2,3 \\
-1 & \text { for } i=4,5,6 \\
-3 & \text { for } i=7 \\
3 & \text { for } i=8
\end{aligned}\right.\right.
$$

where $\left\{\tilde{\mathcal{X}}_{1}, \cdots, \tilde{\chi}_{8}\right\}=\operatorname{Irr}\left(b_{0}\right)$. As in the proof of Lemma 5.3 , using the generalized decomposition numbers of $B_{0}$ relative to $z$,

$$
\chi_{i}(z)= \begin{cases} \pm 1 & \text { for } i=1, \cdots, 6 \\ \pm 3 & \text { for } i=7,8\end{cases}
$$

where $\left\{\chi_{1}, \cdots, \chi_{8}\right\}=\operatorname{Irr}\left(B_{0}\right)$. Since $|G: L|$ is an odd prime, $I_{G}\left(\tilde{\chi}_{7}\right)=I_{G}\left(\tilde{\chi}_{8}\right)=G$ from $(* *)$. Thus, by Proposition 1.4, Clifford's theorem, $\left(^{* *}\right)$ and $(* * *)$, we may assume that $\left.\chi_{7}\right|_{L}=\tilde{\chi}_{7}$ and $\left.\chi_{8}\right|_{L}=\tilde{\chi}_{8}$. By Clifford's theorem, Proposition 1.3, (***) and (**), we have $\left.\chi_{i}\right|_{L} \in \operatorname{Irr}\left(b_{0}\right)$ for $i=1, \cdots, 6$. Thus, by Proposition 1.4, we may assume that $\left.\chi_{i}\right|_{L}=\tilde{\chi}_{i}$ for $i=1, \cdots, 6$. These show $I_{G}\left(\tilde{\chi}_{j}\right)=G$ for all $\tilde{\chi}_{j} \in \operatorname{Irr}\left(b_{0}\right)$. By Proposition 5.1(3), $k\left(B_{0}\right)=k\left(b_{0}\right)$ and $l\left(B_{0}\right)=l\left(b_{0}\right)$. Thus, $B_{0} \cong b_{0}$ from Corollary 1.7. Then, $(*)$ is proved. Since $G / S$ is solvable by $[12$, Theorem], by repeating the above way, we can prove (ii).

REMARK 1. If $G$ is solvable, we easily know $B_{0}$ since we may assume $O(G)=1$ from Lemma 1.1. Assume $G$ is nonsolvable. If $e=3$ or 7 , we know $B_{0}$ from Theorem 2.4. If $e=21$, we know $B_{0}$ from Theorems 4.8 and 5.4.

REMARK 2. By Remark 1 of $\S 2$, there is a finite group $G$ with elementary abelian Sylow 2-subgroups of order 8 such that $e(G)=21$ and $e(S)=7$ where $S=O^{\prime}(G / O(G))$. 


\section{The case when $P$ is elementary abelian of order $\mathbf{1 6}$}

In this section we deal with the case when $G$ has elementary abelian Sylow 2-subgroups of order 16 . Specially, we are interested in the case where $e$ is not prime. When $e$ is 9 or 21, the similar phenomenon to Theorem 5.4 occurs. Throughout this section we assume that $G$ has an elementary abelian Sylow 2-subgroup $P$ of order 16 and we use the notation $e$ and $B_{0}$ as usual.

By Lemma 1.13 and Remark 1 of $\S 1$, it is enough to consider the cases when $e=3,5,7,9,15$ and 21 .

Proposition 6.1. If $G$ is solvable and $e=3$, then one of the following holds.

(i) $B_{0} \cong B_{0}(M)$ where $M$ is a semi-direct product of its normal subgroup $P$ by $\langle t\rangle$ such that $P=\langle x, y, z, w\rangle$ is elementary abelian of order $16,\langle t\rangle$ is cyclic of order $3, x^{t}=y, y^{t}=x y, z^{t}=w$ and $w^{t}=z w$. In this case $k\left(B_{0}\right)=8$.

(ii) $B_{0} \cong B_{0}(L)$ where $L$ is a semi-direct product of its normal subgroup $P$ by $\langle t\rangle$ such that $P=\langle x, y, z, w\rangle$ is elementary abelian of order $16,\langle t\rangle$ is cyclic of order $3, x^{t}=x, y^{t}=y, z^{t}=w$ and $w^{t}=z w$. In this case $k\left(B_{0}\right)=16$.

Proof. By Lemma 1.1, we may assume $O(G)=1$. Hence $G$ is a semidirect product of its normal subgroup $P$ by $Z_{3}$ and $G$ is not the direct product $P \times Z_{3}$. Let $G=P\langle t\rangle$ where $\langle t\rangle$ is cyclic of order 3 , and let $P=\langle x, y, z, w\rangle$. We may assume that

(i) $x^{t}=y, y^{t}=x y, z^{t}=w, w^{t}=z w$

or

(ii) $x^{t}=x, y^{t}=y, z^{t}=w, w^{t}=z w$.

Then we can easily prove the assertion.

Proposition 6.2. Let $D$ be the decomposition matrix of $B_{0}$. If $e=3$, then we have the following.

(i) When $G$ is solvable, $D$ has the form

$\begin{array}{llllll}1 & 0 & 0 & 1 & 0 & 0 \\ 0 & 1 & 0 & 1 & 0 & 0 \\ 0 & 0 & 1 & 1 & 0 & 0 \\ 1 & 1 & 1 & 1 & 0 & 0 \\ 1 & 1 & 1 & 0 & 1 & 0 \\ 1 & 1 & 1 & 0 & 1 & 0 \\ 1 & 1 & 1 & 0 & 1 & 0 \\ 1 & 1 & 1, \text { or } & 0 & 1 & 0 \\ k\left(B_{0}\right)=8 & 0 & 0 & 1\end{array}$




$\begin{array}{lll}0 & 0 & 1 \\ 0 & 0 & 1 \\ 0 & 0 & 1 \\ 1 & 1 & 1 \\ 1 & 1 & 1 \\ 1 & 1 & 1 \\ 1 & 1 & 1 \\ k\left(B_{0}\right)=16\end{array}$

(ii) When $G$ is nonsolvable, we obtain $D$ from Theorem 2.4(2) and Lemma 1.16(ii).

Proof. The assertion is proved by Proposition 6.1.

Proposition 6.3. If $e=5$, then $G$ is solvable, $B_{0} \cong B_{0}\left(P \cdot Z_{5}\right)$ where $P \cdot Z_{5}$ is the semi-direct product of its normal subgroup $P$ by $Z_{5}$ and it is not the direct product $P \times Z_{5}$, and the decomposition matrix of $B_{0}$ has the form

$\begin{array}{lllll}1 & 0 & 0 & 0 & 0 \\ 0 & 1 & 0 & 0 & 0 \\ 0 & 0 & 1 & 0 & 0 \\ 0 & 0 & 0 & 1 & 0 \\ 0 & 0 & 0 & 0 & 1 \\ 1 & 1 & 1 & 1 & 1 \\ 1 & 1 & 1 & 1 & 1 \\ 1 & 1 & 1 & 1 & 1 .\end{array}$

Proof. By Proposition 1.10 and Lemma 1.12, $G$ is solvable since we may assume $O(G)=1$ by Lemma 1.1. Hence $G$ is the semi-direct product of $P$ by $Z_{5}$, and it is not the direct product $P \times Z_{5}$. The decomposition matrix of $B_{0}$ is easily obtained.

Proposition 6.4. If $e=7$, then there is a basic set $W$ of $B_{0}$ such that $W$ contains the trivial Brauer character and the decomposition matrix of $B_{0}$ with respect to $W$ has the form 


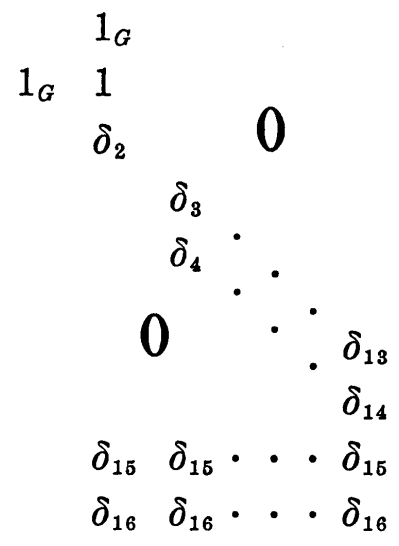

where $\delta_{i}= \pm 1$.

Proof. As in the proof of Lemma 4.1 we can prove the assertion by Proposition 5.2 .

PROPOSITION 6.5. Suppose $k\left(B_{0}\right)=16$.

(1) If $G$ has an involution $x$ with $b_{x} \cong B_{0}\left(P \cdot Z_{3}\right)$ where $P \cdot Z_{3}$ is a semidirect product of $P$ by $Z_{3}$ and it is not the direct product $P \times Z_{3}$, then the generalized decomposition matrix $D^{x}$ of $B_{0}$ relative to $x$ has the form $\left(^{*}\right)$.

(2) If $G$ has an involution $x$ with $b_{x} \cong B_{0}\left(Z_{2} \times Z_{2} \times L_{2}(q)\right)$ for some $q>3$ with $q \equiv 3$ or $5(\bmod 8)$, then the generalized decomposition matrix $D^{x}$ of $B_{0}$ relative to $x$ is as follows:

(i) When $3<q \equiv 3(\bmod 8), D^{x}$ has the form $\left(^{*}\right)$.

(ii) When $3<q \equiv 5(\bmod 8), D^{x}$ has the form $(* *)$.

\begin{tabular}{|c|c|c|c|c|c|c|}
\hline \multirow{2}{*}{\multicolumn{3}{|c|}{$\begin{array}{lll} & 1_{M} & \\
1_{G} & 1 & 0\end{array}$}} & \multicolumn{4}{|c|}{$1_{M}$} \\
\hline & & & $1_{G}$ & 1 & 0 & 0 \\
\hline$\delta_{2}$ & 0 & 0 & & $\delta_{2}$ & 0 & 0 \\
\hline$\delta_{3}$ & 0 & 0 & & $\delta_{3}$ & 0 & 0 \\
\hline$\delta_{4}$ & 0 & 0 & & $\delta_{4}$ & 0 & 0 \\
\hline 0 & $\delta_{5}$ & 0 & & $\delta_{5}$ & $\delta_{5}$ & 0 \\
\hline 0 & $\delta_{6}$ & 0 & & $\delta_{6}$ & $\delta_{6}$ & 0 \\
\hline 0 & $\delta_{7}$ & 0 & & $\delta_{7}$ & $\delta_{7}$ & 0 \\
\hline 0 & $\delta_{8}$ & 0 & & $\delta_{8}$ & $\delta_{8}$ & 0 \\
\hline 0 & 0 & $\delta_{9}$ & & $\delta_{9}$ & 0 & $\delta_{9}$ \\
\hline 0 & 0 & $\delta_{10}$ & & $\delta_{10}$ & 0 & $\delta_{10}$ \\
\hline 0 & 0 & $\delta_{11}$ & & $\delta_{11}$ & 0 & $\delta_{11}$ \\
\hline 0 & 0 & $\delta_{12}$ & & $\delta_{12}$ & 0 & $\delta_{12}$ \\
\hline$\delta_{13}$ & $\delta_{13}$ & $\delta_{13}$ & & $\delta_{13}$ & $\delta_{13}$ & $\delta_{13}$ \\
\hline$\delta_{14}$ & $\delta_{14}$ & $\delta_{14}$ & & $\delta_{14}$ & $\delta_{14}$ & $\delta_{14}$ \\
\hline
\end{tabular}




$$
\begin{array}{lll}
\delta_{15} & \delta_{15} & \delta_{15} \\
\delta_{16} & \delta_{16} & \delta_{16}
\end{array}
$$

$$
\begin{array}{lll}
\delta_{15} & \delta_{15} & \delta_{15} \\
\delta_{16} & \delta_{16} & \delta_{16}
\end{array}
$$

where $\delta_{i}= \pm 1$ and $M=C_{G}(x)$.

Proof. (1) By Proposition 6.2(i), we know the Cartan matrix of $b_{x}$. Hence the assertion is proved as in the proof of Lemma 4.1 .

(2) We obtain the Cartan matrix of $b_{x}$ from Lemma 1.16(ii). Thus we can verify (2) as in the proof of (1).

LEMMA 6.6. Assume $e=9, O(G)=1, O^{\prime}(G)=Z_{2} \times Z_{2} \times L_{2}(q)$ for some $q>3$ with $q \equiv 3$ or $5(\bmod 8)$, and $G$ has a normal subgroup $H$ of odd prime index with $e(H)=3$. Let $b_{0}=B_{0}(H)$, and let $x$ and $z$ be involutions in $Z\left(O^{\prime}(G)\right.$ ) and $L_{2}(q)$, respectively. Then we have the following.

(i) $\left.\chi_{i}\right|_{H}=\left.\chi_{i+1}\right|_{H}=\left.\chi_{i+2}\right|_{H}=\tilde{\chi}_{i}$ for $i=1,5,9,13$

$$
\left.\chi_{j}\right|_{H}=\tilde{\chi}_{j-2}+\tilde{\chi}_{j-1}+\tilde{\chi}_{j} \text { for } j=4,8,12,16
$$

and the values $\tilde{\chi}_{i}(1), \tilde{\chi}_{i}(x), \tilde{\chi}_{i}(z)$ and $\tilde{\chi}_{i}(x z)$ are as follows:

$\begin{array}{llcrrr} & 1 & x & z & x z \\ \tilde{\chi}_{1}, \tilde{\chi}_{2} & 1 & 1 & 1 & 1 \\ \tilde{\chi}_{3}, \tilde{\chi}_{4} & 1 & -1 & 1 & -1 \\ \tilde{\chi}_{5}, \tilde{\chi}_{6} & (q+\varepsilon) / 2 & (q+\varepsilon) / 2 & -\varepsilon & -\varepsilon \\ \tilde{\chi}_{7}, \tilde{\chi}_{8} & (q+\varepsilon) / 2 & -(q+\varepsilon) / 2 & -\varepsilon & \varepsilon \\ \tilde{\chi}_{9}, \tilde{\chi}_{10} & (q+\varepsilon) / 2 & (q+\varepsilon) / 2 & -\varepsilon & -\varepsilon \\ \tilde{\chi}_{11}, \tilde{\chi}_{12} & (q+\varepsilon) / 2 & -(q+\varepsilon) / 2 & -\varepsilon & \varepsilon \\ \tilde{\chi}_{13}, \tilde{\chi}_{14} & q & q & \varepsilon & \varepsilon \\ \tilde{\chi}_{15}, \tilde{\chi}_{16} & q & -q & \varepsilon & -\varepsilon\end{array}$

where $\left\{\chi_{1}=1_{G}, \chi_{2}, \cdots, \chi_{16}\right\}=\operatorname{Irr}\left(B_{0}\right),\left\{\tilde{\chi}_{1}=1_{H}, \tilde{\chi}_{2}, \cdots, \tilde{\chi}_{16}\right\}=\operatorname{Irr}\left(b_{0}\right)$ and $\varepsilon=-1$ if $q \equiv 3$ $(\bmod 8) ; \varepsilon=1$ if $q \equiv 5(\bmod 8)$.

(ii) $\left.\phi_{i}\right|_{H}=\left.\phi_{i+1}\right|_{H}=\left.\phi_{i+2}\right|_{H}=\tilde{\phi}_{(i+2) / 3}$ for $i=1,4,7$

$$
\tilde{\phi}_{j / 3}^{G}=\phi_{j-2}+\phi_{j-1}+\phi_{j} \text { for } j=3,6,9
$$

where $\left\{\phi_{1}=1_{G}, \phi_{2}, \cdots, \phi_{9}\right\}=\operatorname{IBr}\left(B_{0}\right)$ and $\left\{\tilde{\phi}_{1}=1_{H}, \tilde{\phi}_{2}, \tilde{\phi}_{3}\right\}=\operatorname{IBr}\left(b_{0}\right)$.

Proof. Let $S=O^{\prime}(G)=\langle x, y\rangle \times L_{2}(q)$ and $P=\langle x, y, z, w\rangle$ where $\langle z, w\rangle$ is a 2-Sylow subgroup of $L_{2}(q)$. We can write $N_{S}(P)=\left\langle s, C_{S}(P)\right\rangle$ for some $s \in N_{S}(P)$. We may assume $z^{s}=w$ and $w^{s}=z w$. We can also write $N_{G}(P)=\left\langle s, t, C_{G}(P)\right\rangle$ for some $t \in N_{G}(P)$ where $s$ and $t$ have order 3 modulo $C_{G}(P)$ since $e=9$ (cf. Lemma 1.13). We may assume $x^{t}=y, y^{t}=x y, z^{t}=z$ and $w^{t}=w$. As in the proof of Lemma 5.3 , we get $G / H=\langle t H\rangle, C_{G}(P)=C_{H}(P)$ and $|G: H|=3$. By $[10$, Lemma 
18.5], $\{1, x, z, x z\}$ is the set of all representatives of $G$-conjugate classes of $P$. As before, $l\left(b_{x}\right)=l\left(b_{z}\right)=3$ and $l\left(b_{x z}\right)=1$. By Lemma 3.1, $k\left(B_{0}\right)=16$. Since $S$ is normal in $C_{G}(x)$ and $e\left(C_{G}(x)\right)=3$, it follows from Lemma 2.3 that $b_{x} \cong B_{0}\left(Z_{2} \times Z_{2}\right.$ $\left.\times L_{2}(q)\right)$. Thus, by Lemmas 1.16(i) and 6.5(2), we may assume

$$
\chi_{i}(x)= \begin{cases} \pm 1 & \text { for } i=1, \cdots, 4 \\ \pm(q+\varepsilon) / 2 & \text { for } i=5, \cdots, 12 \\ \pm q & \text { for } i=13, \cdots, 16 .\end{cases}
$$

Since $e(H)=3$, by Lemma $2.3, b_{0} \cong B_{0}(S)$. Let $C_{G}(P)=P \times V$. By [10, Theorem 18.4], $P \cap G^{\prime}=P$, so that $\left|G: V G^{\prime}\right|$ is odd. Since $b_{0} \cong B_{0}(S)$ and since $C_{H}(P)=$ $P \times V$, by Proposition $1.5,\left|H: V H^{\prime}\right|=4$. Thus, $\left|G: V G^{\prime}\right|=3$, so that $k^{\prime}\left(B_{0}\right)=3$ from Proposition 1.5. Since $b_{0} \cong B_{0}(S)$, by [10, Theorem 38.1], we know the values of $\left.\tilde{\chi}_{i}\right|_{s}$ for all $i$. Then we get the table in (i). Using this we may assume that

$$
\left.\begin{array}{l}
I_{G}\left(\tilde{\chi}_{i}\right)=G \text { for } i=1,5,9,13 \\
I_{G}\left(\tilde{\chi}_{j-2}\right)=I_{G}\left(\tilde{\chi}_{j-1}\right)=I_{G}\left(\tilde{\chi}_{j}\right)=H \\
\tilde{\chi}_{j-2}^{t}=\tilde{\chi}_{j-1}, \tilde{\chi}_{j-1}^{t}=\tilde{\chi}_{j}
\end{array}\right\} \text { for } j=4,8,12,16 .
$$

By $\left(^{*}\right)$ and $\left(^{* *}\right)$, we may assume that $\left.\chi_{1}\right|_{H}=\left.\chi_{2}\right|_{H}=\left.\chi_{3}\right|_{H}=\tilde{\chi}_{1}$. Since $\tilde{\chi}_{2}(x)+\tilde{\chi}_{3}(x)$ $+\tilde{\chi}_{4}(x)=-1$, by Proposition 1.4, $\left(^{*}\right)$ and $(* *)$, we get $\left.\chi_{4}\right|_{H}=\tilde{\chi}_{2}+\tilde{\chi}_{3}+\tilde{\chi}_{4}$. Similarly, we may assume that $\left.\chi_{j}\right|_{H}=\tilde{\chi}_{j-2}+\tilde{\chi}_{j-1}+\tilde{\chi}_{j}$ for $j=8,12,16$. We may also assume that $\left.\chi_{i}\right|_{H}=\left.\chi_{i+1}\right|_{H}=\left.\chi_{i+2}\right|_{H}=\tilde{\chi}_{i}$ for $i=5,9,13$ using Frobenius reciprocity (*) and ${ }^{* *}$. This completes the proof of (i). Since $b_{0} \cong B_{0}(S)$, by Lemma 1.16(i), $\tilde{\phi}_{2}(1)$ $=\tilde{\phi}_{3}(1)=(q-1) / 2$. Thus $I_{G}\left(\tilde{\phi}_{j}\right)=G$ for $j=1,2,3$ since $|G: H|=3$. For all $\phi_{i} \in \operatorname{IBr}\left(B_{0}\right)$ we have $\left.\phi_{i}\right|_{H} \in \operatorname{IBr}\left(b_{0}\right)$ by Clifford's theorem since $|G: H|=3$. Thus, by $[15, \mathrm{~V} 16.6 \mathrm{Satz}]$, we get (ii) for suitable indexing of $\phi_{2}, \cdots, \phi_{9}$. This completes the proof of the lemma.

Proposition 6.7. Assume as in Lemma 6.6. Then the decomposition matrix $D$ of $B_{0}$ is as follows. 
(i) $3<q \equiv 3(\bmod 8)$ :

$$
\begin{aligned}
& \begin{array}{lll}
1 & 0 & 0
\end{array} \\
& \begin{array}{lll}
0 & 1 & 0
\end{array} \\
& \begin{array}{lll}
0 & 0 & 1
\end{array} \\
& 0 \quad 0 \\
& \begin{array}{lll}
1 & 1 & 1
\end{array} \\
& \begin{array}{lll}
1 & 0 & 0
\end{array} \\
& \begin{array}{lllll}
\mathbf{0} & 0 & 1 & 0 & \\
& 0 & 0 & 1 & 0
\end{array} \\
& D=\quad \begin{array}{llllllll}
1 & 1 & 1 & & & \\
& & & & & 1 & 0 & 0
\end{array} \\
& \begin{array}{lllll}
0 & 0 & 0 & 1 & 0
\end{array} \\
& \begin{array}{lll}
1 & 1 & 1
\end{array} \\
& \begin{array}{lllllllll}
1 & 0 & 0 & 1 & 0 & 0 & 1 & 0 & 0
\end{array} \\
& \begin{array}{lllllllll}
0 & 1 & 0 & 0 & 1 & 0 & 0 & 1 & 0
\end{array} \\
& \begin{array}{lllllllll}
0 & 0 & 1 & 0 & 0 & 1 & 0 & 0 & 1
\end{array} \\
& \begin{array}{lllllllll}
1 & 1 & 1 & 1 & 1 & 1 & 1 & 1 & 1 .
\end{array}
\end{aligned}
$$

(ii) $3<q \equiv 5(\bmod 8)$ :

$$
D=\begin{array}{rrrrrrrrr}
1 & 0 & 0 & & & & & & \\
0 & 1 & 0 & 0 & & & 0 & \\
0 & 0 & 1 & & & & & \\
1 & 1 & 1 & & & & & \\
1 & 0 & 0 & 1 & 0 & 0 & & & \\
0 & 1 & 0 & 0 & 1 & 0 & & \\
0 & 0 & 1 & 0 & 0 & 1 & 0 & \\
1 & 1 & 1 & 1 & 1 & 1 & & & \\
1 & 0 & 0 & & & & 1 & 0 & 0 \\
0 & 1 & 0 & & & & 0 & 1 & 0 \\
0 & 0 & 1 & & & & 0 & 0 & 1 \\
1 & 1 & 1 & & & 1 & 1 & 1 \\
1 & 0 & 0 & & & 1 & 0 & 0 \\
0 & 1 & 0 & (*) & & 0 & 1 & 0 \\
0 & 0 & 1 & & & 0 & 0 & 1 \\
1 & 1 & 1 & 1 & 1 & 1 & 1 & 1 & 1,
\end{array}
$$

where $\left(^{*}\right)$ is one of the following types

$$
\begin{array}{llllllllllllllllll}
1 & 0 & 0 & 1 & 0 & 0 & 0 & 1 & 0 & 0 & 1 & 0 & 0 & 0 & 1 & 0 & 0 & 1 \\
0 & 1 & 0 & 0 & 0 & 1 & 1 & 0 & 0 & 0 & 0 & 1 & 1 & 0 & 0 & 0 & 1 & 0 \\
0 & 0 & 1, & 0 & 1 & 0, & 0 & 0 & 1, & 1 & 0 & 0, & 0 & 1 & 0, & 1 & 0 & 0 .
\end{array}
$$


Proof. We use the same notation as in Lemma 6.6. Let $\tilde{D}$ be the decomposition matrix of $b_{0}$, and let $D=\left(d_{j \lambda}\right)_{j, \lambda}$. Let $\left.\chi_{j}\right|_{H}=\sum_{i} l_{i j} \tilde{\chi}_{i}$ for each $j$, and $L=\left(l_{i j}\right)_{i, j}$. Similarly, let $\tilde{\phi}_{\kappa}^{G}=\sum_{\lambda} \beta_{\kappa \lambda} \phi_{\lambda}$ for each $\kappa$, and $B=\left(\beta_{\kappa \lambda}\right)_{\kappa, \lambda}$. By $[7, \S 26]$, (1)

$$
\widetilde{D} B=L D \text {. }
$$

(i) Since $b_{0} \cong B_{0}(S)$, by (1) and Lemmas 1.16 (ii) and 6.6,

$$
\begin{array}{lll}
1 & 0 & 0 \\
0 & 1 & 0 \\
0 & 0 & 1 \\
1 & 1 & 1
\end{array}
$$$$
0 \quad 0
$$

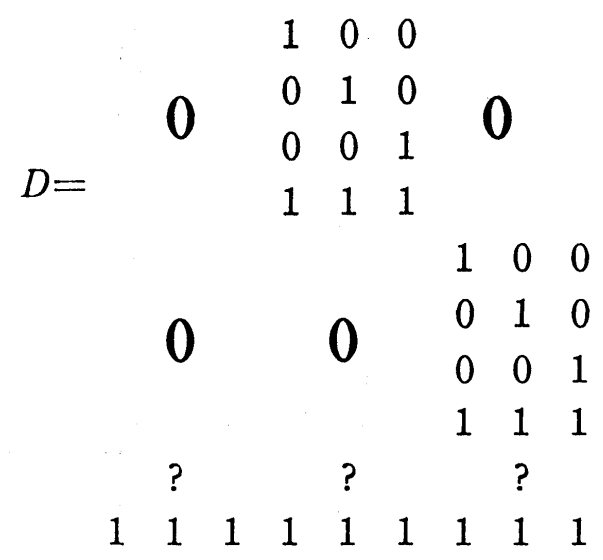

and

(3)

$$
d_{13, \lambda}+d_{14, \lambda}+d_{15, \lambda}=1 \text { for all } \lambda=1, \cdots, 9 .
$$

By Lemma 6.6,

(4)

$$
\begin{array}{r}
1 \\
1 \\
1 \\
-1 \\
1 \\
1 \\
D^{x z}=\left(d_{i 1}^{x z}\right)_{i}= \\
1 \\
-1 \\
1 \\
1 \\
1 \\
-1 \\
-1 \\
-1 \\
-1 \\
1
\end{array}
$$


where $D^{x z}$ is the generalized decomposition matrix of $B_{0}$ relative to $x z$. Clearly, $S$ is normal in $C_{G}(x)$. By the proof of Lemma 3.1, $e\left(C_{G}(x)\right)=3$. Since $q \equiv 3$ (mod. $8)$, by Lemmas 2.3 and $6.5(2)$,

$$
\begin{aligned}
& \phi_{1}^{x} \quad \phi_{2}^{x} \quad \phi_{3}^{x} \\
& 1_{G}=\chi_{\nu_{1}} \quad 1 \quad 0 \quad 0 \\
& \begin{array}{llll}
\chi_{\nu_{2}} & \delta_{2} & 0 & 0
\end{array} \\
& \begin{array}{llll}
\chi_{\nu_{3}} & \delta_{3} & 0 & 0
\end{array} \\
& \begin{array}{llll}
\chi_{\nu_{4}} & \delta_{4} & 0 & 0
\end{array} \\
& \begin{array}{llll}
\chi_{\nu_{5}} & 0 & \delta_{5} & 0
\end{array} \\
& \begin{array}{llll}
\chi_{\nu_{6}} & 0 & \delta_{6} & 0
\end{array} \\
& \begin{array}{llll}
\chi_{\nu_{7}} & 0 & \delta_{7} & 0
\end{array} \\
& \left(d_{\nu, \alpha, \alpha}^{x}\right)_{i, \alpha}=\begin{array}{llll}
\chi_{\nu_{8}} & 0 & \delta_{8} & 0
\end{array} \\
& \begin{array}{llll}
\chi_{\nu 9} & 0 & 0 & \delta_{9}
\end{array} \\
& \begin{array}{llll}
\chi_{\nu_{10}} & 0 & 0 & \delta_{10}
\end{array} \\
& \begin{array}{llll}
\chi_{\nu_{11}} & 0 & 0 & \delta_{11}
\end{array} \\
& \begin{array}{llll}
\chi_{\nu_{12}} & 0 & 0 & \delta_{12}
\end{array} \\
& \begin{array}{llll}
\chi_{\nu_{13}} & \delta_{13} & \delta_{13} & \delta_{13}
\end{array} \\
& \begin{array}{llll}
\chi_{\nu_{14}} & \delta_{14} & \delta_{14} & \delta_{14}
\end{array} \\
& \begin{array}{llll}
\chi_{\nu_{15}} & \delta_{15} & \delta_{15} & \delta_{15}
\end{array} \\
& \begin{array}{llll}
\chi_{\nu_{16}} & \delta_{16} & \delta_{16} & \delta_{16}
\end{array}
\end{aligned}
$$

where $d_{i \alpha}^{x}$ are the generalized decomposition numbers of $B_{0}$ relative to $x, \delta_{i}= \pm 1$, $\chi_{\nu_{1}}=\chi_{1}=1_{G}, \quad\left\{\chi_{\nu_{2}}, \cdots, \chi_{\nu_{16}}\right\}=\left\{\chi_{2}, \cdots, \chi_{16}\right\}, \quad\left\{\phi_{1}^{x}=1_{M}, \phi_{2}^{x}, \phi_{3}^{x}\right\}=\operatorname{IBr}\left(b_{x}\right)$ and $M=C_{G}(x)$. Let $c=\left(\begin{array}{ll}1 & 0 \\ 1 & 1\end{array}\right)$ in $L_{2}(q)$. Then, by [10, Theorem 38.1] and Lemma 1.16, we may assume that

$$
\phi_{2}^{x}(c)=(-1+\sqrt{ }-q) / 2 \text { and } \phi_{3}^{x}(c)=(-1-\sqrt{ }-\bar{q}) / 2 .
$$

By Lemma 6.6 and (5), $\left\{\chi_{\nu_{1}}, \cdots, \chi_{\nu_{4}}\right\}=\left\{\chi_{1}, \cdots, \chi_{4}\right\}$ and $\left\{\chi_{\nu_{13}}, \cdots, \chi_{\nu_{16}}\right\}=\left\{\chi_{13}, \cdots, \chi_{16}\right\}$. We may assume that $\chi_{5} \in\left\{\chi_{\nu_{5}}, \cdots, \chi_{\nu_{8}}\right\}$. By Lemma 6.6, $\left.\chi_{5}\right|_{H}=\left.\chi_{6}\right|_{H}=\left.\chi_{7}\right|_{H}$. Thus, by (5) and (6), we get that $\chi_{6}$ and $\chi_{7}$ are both in $\left\{\chi_{\nu_{5}}, \cdots, \chi_{\nu_{8}}\right\}$. Similarly, none of $\left\{\chi_{9}, \chi_{10}, \chi_{11}\right\}$ are in $\left\{\chi_{\nu_{5}}, \cdots, \chi_{\nu_{8}}\right\}$. Hence, by (2), (5) and (6), we know $\left\{\chi_{\nu_{5}}, \cdots\right.$, $\left.\chi_{\nu 8}\right\}=\left\{\chi_{5}, \cdots, \chi_{8}\right\}$. Thus, $\left\{\chi_{\nu 9}, \cdots, \chi_{\nu_{12}}\right\}=\left\{\chi_{9}, \cdots, \chi_{12}\right\}$. Hence we may assume that $\chi_{\nu_{i}}=\chi_{i}$ for all $i=1, \cdots, 16$. Therefore, by Lemma 6.6,

$\begin{array}{rcl}\phi_{1}^{x} & \phi_{2}^{x} & \phi_{3}^{x} \\ 1 & 0 & 0 \\ 1 & 0 & 0 \\ 1 & 0 & 0 \\ -1 & 0 & 0\end{array}$




$$
\left(d_{i \alpha}^{x}\right)_{i, \alpha}=\begin{array}{rrr}
0 & 1 & 0 \\
0 & 1 & 0 \\
0 & 1 & 0 \\
0 & -1 & 0 \\
0 & 0 & 1 \\
0 & 0 & 1 \\
0 & 0 & 1 \\
0 & 0 & -1 \\
1 & 1 & 1 \\
1 & 1 & 1 \\
1 & 1 & 1 \\
-1 & -1 & -1
\end{array}
$$

Next, we want to know the generalized decomposition numbers $d_{i \alpha}^{z}$ of $B_{0}$ relative to $z$. Let $L=C_{G}(z)$. As for $x, e(L)=3$ and $l\left(B_{0}(L)\right)=3$. Since $N_{L}(P)=\left\langle t, C_{L}(P)\right\rangle$ and $z^{t}=z$, we get from Proposition 6.1 and Theorem 2.4(1) that $k\left(B_{0}(L)\right)=k\left(b_{z}\right)$ $=16$. By Theorem 2.4(2) and Lemmas 6.6 and $6.5, L$ is solvable, so that $b_{z} \cong B_{0}\left(P \cdot Z_{3}\right)$ from Proposition 6.1 where $P \cdot Z_{3}$ is a semi-direct product of its normal subgroup $P$ by $Z_{3}$ and it is not the direct product $P \times Z_{3}$. Thus, by Lemma 6.5,

(8)

$$
\begin{aligned}
& \phi_{1}^{z} \quad \phi_{2}^{z} \quad \phi_{3}^{z} \\
& 1_{G}=\chi_{\nu_{1}} \quad 1 \quad 0 \quad 0 \\
& \begin{array}{llll}
\chi_{\nu_{2}} & 0 & \delta_{2} & 0
\end{array} \\
& \begin{array}{llll}
\chi_{\nu_{3}} & 0 & 0 & \delta_{3}
\end{array} \\
& \begin{array}{llll}
\chi_{\nu_{4}} & \delta_{4} & \delta_{4} & \delta_{4}
\end{array} \\
& \begin{array}{llll}
\chi_{\nu_{5}} & \delta_{5} & 0 & 0
\end{array} \\
& \begin{array}{llll}
\chi_{\nu_{6}} & 0 & \delta_{6} & 0
\end{array} \\
& \begin{array}{lllll}
\chi_{\nu_{7}} & 0 & 0 & \delta_{7}
\end{array} \\
& \left(d_{\nu_{i}, \alpha}^{z}\right)_{i, \alpha}=\begin{array}{llll}
\chi_{\nu_{8}} & \delta_{8} & \delta_{8} & \delta_{8}
\end{array} \\
& \begin{array}{llll}
\chi_{\nu_{9}} & \delta_{9} & 0 & 0
\end{array} \\
& \begin{array}{llll}
\chi_{\nu_{10}} & 0 & \delta_{10} & 0
\end{array} \\
& \begin{array}{llll}
\chi_{\nu_{11}} & 0 & 0 & \delta_{11}
\end{array} \\
& \begin{array}{llll}
\chi_{\nu_{12}} & \delta_{12} & \delta_{12} & \delta_{12}
\end{array} \\
& \begin{array}{llll}
\chi_{\nu_{13}} & \delta_{13} & 0 & 0
\end{array} \\
& \begin{array}{llll}
\chi_{\nu_{14}} & 0 & \delta_{14} & 0
\end{array} \\
& \begin{array}{llll}
\chi_{\nu_{15}} & 0 & 0 & \delta_{15}
\end{array} \\
& \begin{array}{llll}
\chi_{\nu_{16}} & \delta_{16} & \delta_{16} & \delta_{16}
\end{array}
\end{aligned}
$$


where $\delta_{i}= \pm 1, \quad \chi_{\nu_{1}}=\chi_{1}=1_{G}, \quad\left\{\chi_{\nu_{2}}, \cdots, \chi_{\nu_{16}}\right\}=\left\{\chi_{2}, \cdots, \chi_{16}\right\}$ and $\left\{\phi_{1}^{z}, \phi_{2}^{z}, \phi_{3}^{z}\right\}=\operatorname{IBr}\left(b_{2}\right)$. Clearly, $\phi_{1}^{z}(1)=\phi_{2}^{z}(1)=\phi_{3}^{z}(1)=1$. Hence, by Lemma 6.6 and (8),

(9)

$$
\left\{\chi_{\nu_{4}}, \chi_{\nu_{8}}, \chi_{\nu_{12}}, \chi_{\nu_{16}}\right\}=\left\{\chi_{4}, \chi_{8}, \chi_{12}, \chi_{16}\right\}
$$

$$
\left\{\delta_{4}, \delta_{8}, \delta_{12}, \delta_{16}\right\}=\{1,1,1,-1\} \text {. }
$$

By Lemma 6.6, $\chi_{i}(z)=\chi_{i+1}(z)=\chi_{i+2}(z)=1$ for $i=1,5,9$. So it follows from (4), (7),

(8) and [10, Theorem 63.3] that $\delta_{4}=\delta_{8}=\delta_{12}=1$ and $\delta_{16}=-1$. Thus, again by (4),

(7), (8) and [10, Theorem 63.3],

(10)

$$
\begin{aligned}
& \phi_{1}^{z} \quad \phi_{2}^{z} \quad \phi_{3}^{z} \\
& \begin{array}{lll}
1 & 0 & 0
\end{array} \\
& \begin{array}{lll}
0 & 1 & 0
\end{array} \\
& \begin{array}{lll}
0 & 0 & 1
\end{array} \\
& \begin{array}{lll}
1 & 1 & 1
\end{array} \\
& \begin{array}{lll}
1 & 0 & 0
\end{array} \\
& \begin{array}{lll}
0 & 1 & 0
\end{array} \\
& 0 \quad 0 \quad 1 \\
& \left(d_{i \alpha}^{z}\right)_{i, \alpha}=\begin{array}{lll}
1 & 1 & 1 \\
1 & 0 & 0
\end{array} \\
& \begin{array}{lll}
0 & 1 & 0
\end{array} \\
& \begin{array}{lll}
0 & 0 & 1
\end{array} \\
& \begin{array}{lll}
1 & 1 & 1
\end{array} \\
& \begin{array}{lll}
-1 & 0 & 0
\end{array} \\
& \begin{array}{lll}
0 & -1 & 0
\end{array} \\
& \begin{array}{llll}
0 & 0 & -1
\end{array} \\
& \begin{array}{lll}
-1 & -1 & -1
\end{array}
\end{aligned}
$$

for suitable indexing. By (2), (3), (10) and [10, Theorem 63.3],

$$
D=\begin{array}{llllllllll}
\chi_{13} & 1 & 0 & 0 & 1 & 0 & 0 & 1 & 0 & 0 \\
\chi_{14} & 0 & 1 & 0 & 0 & 1 & 0 & 0 & 1 & 0 \\
\chi_{15} & 0 & 0 & 1 & 0 & 0 & 1 & 0 & 0 & 1 \\
\chi_{16} & 1 & 1 & 1 & 1 & 1 & 1 & 1 & 1 & 1 .
\end{array}
$$

This completes the proof of (i).

(ii) Since $b_{0} \cong B_{0}(S)$, as in the proof of (i) we get 
(11)

$$
\begin{aligned}
& \begin{array}{lll}
1 & 0 & 0
\end{array} \\
& \begin{array}{lll}
0 & 1 & 0
\end{array} \\
& \begin{array}{lll}
0 & 0 & 1
\end{array} \\
& 0 \quad 0 \\
& \begin{array}{lll}
1 & 1 & 1
\end{array} \\
& D=\begin{array}{llllll} 
& \text { ? } & & & & \text { ? } \\
1 & 1 & 1 & 1 & 1 & 1
\end{array}
\end{aligned}
$$

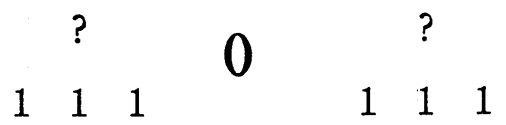

$$
\begin{aligned}
& \text { ? ? ? } \\
& \begin{array}{lllllllll}
1 & 1 & 1 & 1 & 1 & 1 & 1 & 1 & 1
\end{array}
\end{aligned}
$$

and

$$
\begin{array}{ll}
d_{5 \lambda}+d_{6 \lambda}+d_{7 \lambda}=1 & \text { for } \lambda=1, \cdots, 6 \\
d_{9 \lambda}+d_{10, \lambda}+d_{11, \lambda}=1 & \text { for } \lambda=1,2,3,7,8,9 \\
d_{13, \lambda}+d_{14, \lambda}+d_{15, \lambda}=1 & \text { for } \lambda=1, \cdots, 9 .
\end{array}
$$

As in the proof of (i), we have

$$
\begin{array}{rrrrrrr} 
& \phi_{1}^{x} & \phi_{2}^{x} & \phi_{3}^{x} & \phi_{1}^{z} & \phi_{2}^{z} & \phi_{3}^{z} \\
1 & 1 & 0 & 0 & 1 & 0 & 0 \\
1 & 1 & 0 & 0 & 0 & 1 & 0 \\
1 & 1 & 0 & 0 & 0 & 0 & 1 \\
-1 & -1 & 0 & 0 & 1 & 1 & 1 \\
-1 & 1 & 1 & 0 & -1 & 0 & 0 \\
-1 & 1 & 1 & 0 & 0 & -1 & 0 \\
-1 & 1 & 1 & 0 & 0 & 0 & -1 \\
\left(d_{i 1}^{x z}\right)_{i}= & -1 & -1 & 0 & \left(d_{i \alpha}^{z}\right)_{i, \alpha}=-1 & -1 & -1 \\
1 & 1 & 0 & 1 & -1 & 0 & 0 \\
-1 & 1 & 0 & 1 & 0 & -1 & 0 \\
-1 & 1 & 0 & 1 & 0 & 0 & -1 \\
-1 & -1 & 0 & -1 & -1 & -1 & -1 \\
1 & 1 & 1 & 1 & 1 & 0 & 0 \\
1 & 1 & 1 & 1 & 0 & 1 & 0 \\
1 & 1 & 1 & 1 & 0 & 0 & 1 \\
1 & -1 & -1 & -1 & 1 & 1 & 1
\end{array}
$$

$\begin{array}{rrr}\phi_{1}^{z} & \phi_{2}^{z} & \phi_{3}^{z} \\ 1 & 0 & 0 \\ 0 & 1 & 0 \\ 0 & 0 & 1 \\ 1 & 1 & 1 \\ -1 & 0 & 0 \\ 0 & -1 & 0 \\ 0 & 0 & -1 \\ \left(d_{i \alpha}^{z}\right)_{i, \alpha}=-1 & -1 & -1 \\ -1 & 0 & 0 \\ 0 & -1 & 0 \\ 0 & 0 & -1 \\ -1 & -1 & -1 \\ 1 & 0 & 0 \\ 0 & 1 & 0 \\ 0 & 0 & 1 \\ 1 & 1 & 1\end{array}$

where $d_{i 1}^{r z}, d_{i \alpha}^{x}, d_{i \alpha}^{z}, \phi_{\alpha}^{x}$ and $\phi_{\alpha}^{z}$ are the same as in (i). By Lemmas 6.6 and 1.16, we know the degrees of all $\chi_{i}$ and $\phi_{\alpha}$. Thus, by (11), (12), (13) and [10, Theorem 63.3], we may assume 


$$
D=\begin{array}{lllllll}
\chi_{5} & 1 & 0 & 0 & 1 & 0 & 0 \\
\chi_{6} & 0 & 1 & 0 & 0 & 1 & 0 \\
\chi_{7} & 0 & 0 & 1 & 0 & 0 & 1 \\
\chi_{8} & 1 & 1 & 1 & 1 & 1 & 1
\end{array}
$$

Similarly, we may assume

$$
\begin{aligned}
& \begin{array}{llll}
\chi_{9} & 1 & 0 & 0
\end{array} \\
& D=\begin{array}{llllll}
\chi_{10} & ? & 0 & 0 & 1 & 0 \\
\chi_{11} & & & 0 & 0 & 1
\end{array} \\
& \begin{array}{lllllll}
\chi_{12} & 1 & 1 & 1 & 1 & 1 & 1 .
\end{array}
\end{aligned}
$$

\begin{tabular}{|c|c|c|c|c|c|c|c|c|c|c|c|c|c|c|c|c|c|}
\hline$\chi_{9}$ & 1 & 0 & 0 & 1 & 0 & 0 & 0 & 1 & 0 & 0 & 0 & 1 & 0 & 1 & 0 & 0 & 0 \\
\hline$\chi_{10}$ & 0 & 1 & 0 & 0 & 0 & 1 & 1 & 0 & 0 & 1 & 0 & 0 & 0 & 0 & 1 & 0 & 1 \\
\hline$\chi_{11}$ & 0 & 0 & 1 & 0 & 1 & 0 & 0 & 0 & 1 & 0 & 1 & 0 & 1 & 0 & 0 & 1 & 0 \\
\hline$\chi_{12}$ & 1 & 1 & 1 & 1 & 1 & 1 & 1 & 1 & 1 & 1 & 1 & 1 & 1 & 1 & 1 & 1 & 1 \\
\hline$\chi_{13}$ & 1 & 0 & 0 & 1 & 0 & 0 & 0 & 1 & 0 & 0 & 0 & 1 & 0 & 1 & 0 & 0 & 0 \\
\hline$\chi_{14}$ & 0 & 1 & 0 & 0 & 0 & 1 & 1 & 0 & 0 & 1 & 0 & 0 & 0 & 0 & 1 & 0 & 1 \\
\hline$\chi_{15}$ & 0 & 0 & 1 & 0 & 1 & 0 & 0 & 0 & 1 & 0 & 1 & 0 & 1 & 0 & 0 & 1 & 0 \\
\hline$\chi_{16}$ & & 1 & $\begin{array}{l}1 \\
\phi_{3}\end{array}$ & 1 & 1 & 1, & 1 & 1 & 1, & 1 & 1 & 1, & 1 & 1 & $\begin{array}{l}1, \\
\phi_{3}\end{array}$ & 1 & 1 \\
\hline
\end{tabular}

So, by (11), (12), (13), (14), (15) and [10, Theorem 63.3],

$$
\begin{aligned}
& \begin{array}{lllllll}
\chi_{13} & 1 & 0 & 0 & 1 & 0 & 0
\end{array} \\
& D=\chi_{14} \quad \text { ? } \quad \begin{array}{lllllll}
0 & 1 & 0 & 0 & 1 & 0
\end{array} \\
& \begin{array}{lllllll}
\chi_{15} & 0 & 0 & 1 & 0 & 0 & 1
\end{array} \\
& \begin{array}{llllllllll}
\chi_{16} & 1 & 1 & 1 & 1 & 1 & 1 & 1 & 1 & 1 .
\end{array}
\end{aligned}
$$

Thus, considering the degrees of $\chi_{i}$ and $\phi_{\alpha}$, by (11)-(16) we get the following six cases:

Thus, for suitable indexing of $\chi_{i}$ and $\phi_{\alpha}$, we obtain (ii).

The following theorem is one of the main results of this section.

THEOREM 6.8. Let $\bar{G}=G / O(G)$ and $S=O^{\prime}(\bar{G})$. If $G$ is nonsolvable, $e=9$ and $e(S) \neq 9$, then we have the following.

(i) $S \cong Z_{2} \times Z_{2} \times L_{2}$ (q) for some $q>3$ with $q \equiv 3$ or $5(\bmod 8)$.

(ii) For any subnormal subgroup $\bar{L}$ of $\bar{G}$ of odd index with $e(\bar{L})=9$, $B_{0} \cong B_{0}(\bar{L})$.

Proof. We may assume $O(G)=1$ by Lemma 1.1 , so that $S=O^{\prime}(G)$. 
(i) Since $G$ is nonsolvable, $e(S)=3$. Thus, we get (i) from Proposition 1.10 and Lemma 1.12 .

(ii) Firstly, we want to prove that

if $L$ is a normal subgroup of $G$ such that $|G: L|$ is an odd prime and $\left\{\begin{array}{l}e(L)=9 \text { and if } H \text { is a normal subgroup of } L \text { such that }|L: H| \text { is an odd } \\ \text { prime and } e(H)=3 \text {, then } B_{0} \cong B_{0}(L) .\end{array}\right.$

Let $b_{0}=B_{0}(L)$. By Lemma 3.1, $k\left(B_{0}\right)=k\left(b_{0}\right)=16$ and $l\left(B_{0}\right)=l\left(b_{0}\right)=9$. We may write $O^{\prime}(G)=S=\langle x, y\rangle \times L_{2}(q)$ and $P=\langle x, y, z, w\rangle$ where $\langle z, w\rangle$ is a Sylow 2subgroup of $L_{2}(q)$. By the proof of Lemma 6.6, we may assume $x^{s}=x, y^{s}=y$, $z^{s}=w, w^{s}=z w, x^{t}=y, y^{t}=x y, z^{t}=z$ and $w^{t}=w$ where $s, t \in N_{L}(P)=\left\langle s, t, C_{L}(P)\right\rangle$. So $N_{G}(P)=\left\langle s, t, C_{G}(P)\right\rangle$. By the proof of Lemma 3.1, $l\left(b_{x z}\right)=1$. Thus, by Lemma 1.9, $\chi_{i}(x z)= \pm 1$ for all $\chi_{i} \in \operatorname{Irr}\left(B_{0}\right)$. By Lemma 6.6, we know the values $\tilde{\chi}_{j}(1)$ and $\tilde{\chi}_{j}(x z)$ for all $\tilde{\chi}_{j} \in \operatorname{Irr}\left(b_{0}\right)$. Using this, if $\tilde{\chi}, \tilde{\chi}^{\prime} \in \operatorname{Irr}\left(b_{0}\right)$ and $\tilde{\chi}(1)=\tilde{\chi}^{\prime}(1)$, then $\tilde{\chi}(x z)=$ $\tilde{\chi}^{\prime}(x z)= \pm 1$. Hence it follows from Corollary 1.8 that $B_{0} \cong b_{0}$. Thus we get $\left(^{*}\right)$. On the other hand, $G / S$ is solvable from [12, Theorem]. Hence we can verify (ii) by repeating the above way. This completes the proof.

PROPOSITION 6.9. Let $D$ be the decomposition matrix of $B_{0}$, and let $S=$ $O^{\prime}(G / O(G))$. If $e=9$, then we have following.

(i) When $G$ is solvable,

$$
\begin{array}{ccccccccc}
1 & 0 & 0 & & & & & & \\
0 & 1 & 0 & & 0 & & & 0 & \\
0 & 0 & 1 & & & & & & \\
& & & 1 & 0 & 0 & & & \\
& 0 & & 0 & 1 & 0 & & 0 & \\
& & & 0 & 0 & 1 & & & \\
& & & & & & & \\
& & & & & & 1 & 0 & 0 \\
& 0 & & & 0 & & 0 & 1 & 0 \\
& & & & & & 0 & 0 & 1 \\
1 & 1 & 1 & 0 & 0 & 0 & 0 & 0 & 0 \\
0 & 0 & 0 & 1 & 1 & 1 & 0 & 0 & 0 \\
0 & 0 & 0 & 0 & 0 & 0 & 1 & 1 & 1 \\
1 & 0 & 0 & 1 & 0 & 0 & 1 & 0 & 0 \\
0 & 1 & 0 & 0 & 1 & 0 & 0 & 1 & 0 \\
0 & 0 & 1 & 0 & 0 & 1 & 0 & 0 & 1 \\
1 & 1 & 1 & 1 & 1 & 1 & 1 & 1 & 1 .
\end{array}
$$

(ii) When $G$ is nonsolvable and $e(S)=9$, we know $D$ from Theorem $3.4(2)$ 
and Lemma 1.16(ii).

(iii) When $G$ is nonsolvable and $e(S)=3$, we know $D$ from Theorem 6.8 and Proposition 6.7.

REMARK 1. There is a finite group $G$ with an elementary abelian Sylow 2-subgroup $P$ of order 16 such that $e(G)=9$ and $O^{\prime}(G / O(G)) \cong Z_{2} \times Z_{2} \times L_{2}(q)$ for $q>3$ with $q \equiv 3$ or $5(\bmod 8)$. Let $\langle z, w\rangle$ be a Sylow 2-subgroup of $L_{2}(q)$, and let $S=\langle x, y\rangle \times L_{2}(q)$ and $P=\langle x, y, z, w\rangle$ where $\langle x, y\rangle$ is elementary abelian of order 4. There is an automorphism $r$ of $\langle x, y\rangle$ with $x^{r}=y$ and $y^{r}=x y$. We can consider that $r \in \operatorname{Aut}(S)$ if its we consider that $r$ is trivial on $L_{2}(q)$. So there is a semi-direct product $G$ of its normal subgroup $S$ by $\langle r\rangle$. Then, $e(G)=9$ and $O^{\prime}(G)=$ $S=Z_{2} \times Z_{2} \times L_{2}(q)$.

The next theorem is one of the main results of this section.

THEOREM 6.10. If $G$ is nonsolvable and $e=15$, then $B_{0} \cong B_{0}(S L(2,16))$.

Proof. By Lemma 1.1, we may assume $O(G)=1$. Let $S=O^{\prime}(G)$. Since $G$ is nonsolvable, it follows from Proposition 1.10 and Lemma 1.12 that $e(S) \neq 1$ and $e(S) \neq 5$. So that $e(S)=3$ or 15 . Firstly, suppose $e(S)=3$. By Proposition 1.10 and Lemma $1.12, S \cong Z_{2} \times Z_{2} \times L_{2}(q)$ for some $q>3$ with $q \equiv 3$ or $5(\bmod 8)$. Thus, there is an involution $x \in P \cap Z(S)$. We can write $N_{S}(P)=\left\langle s, C_{S}(P)\right\rangle$ for some $s \in N_{S}(P)$. Thus, $x^{s}=x$. Since $e=15$, we can write $N_{G}(P)=\left\langle t, C_{G}(P)\right\rangle$ for some $t \in N_{G}(P)$. Since $N_{S}(P) / C_{S}(P)$ can be considered as a subgroup of $N_{G}(P) / C_{G}(P)$ through the canonical monomorphism, we get that $s \equiv t^{5 i}\left(\bmod C_{G}(P)\right)$ for some integer $i$ with $i \neq 0(\bmod 3)$. Thus, $x=x^{t^{5 i}}$. This is a contradiction. Hence $e(S)=15$, so that $S \cong S L(2,16)$ from Proposition 1.10 and Lemma 1.12 .

We prove $B_{0} \cong B_{0}(S)$ by induction on $|G|$. Let $G \neq S$. Since $G / S$ is solvable by [12, Theorem], $G$ has a normal subgroup $H$ of odd prime index with $S \cong H$. Let $b_{0}=B_{0}(H)$, and let $z$ be an involution in $P$. Since $b_{0} \cong B_{0}(S)$ by induction, we get $k\left(b_{0}\right)=16$ and

$$
\tilde{\chi}_{i}(1)=\left\{\begin{array}{rl}
1 & \text { for } i=1 \\
15 & \text { for } i=2, \cdots, 9 \\
17 & \text { for } i=10, \cdots, 16,
\end{array} \quad \tilde{\chi}_{i}(z)=\left\{\begin{aligned}
1 & \text { for } i=1 \\
-1 & \text { for } i=2, \cdots, 9 \\
1 & \text { for } i=10, \cdots, 16
\end{aligned}\right.\right.
$$

using $\left[10\right.$, Theorem 38.2], where $\left\{\tilde{\chi}_{1}, \cdots, \tilde{\chi}_{16}\right\}=\operatorname{Irr}\left(b_{0}\right)$. Since all involutions in $P$ are $G$-conjugate, $P \cap G^{\prime}=P$ by $\left[10\right.$, Theorem 18.4]. Thus, $k^{\prime}\left(B_{0}\right)$ is odd from Proposition 1.5. Now, we want to claim that $k\left(B_{0}\right)=16$. If $k^{\prime}\left(B_{0}\right)=1$, we get from Propositions 1.5 and 1.6 that $k\left(B_{0}\right)=16$. Suppose $k\left(B_{0}\right) \neq 16$. Since $e=15$, 
$l\left(b_{z}\right)=1$. Thus, by Lemma $1.15(2), k\left(B_{0}\right)=8$. So that $k^{\prime}\left(B_{0}\right)=3,5$ or 7 . Let $\left\{\chi_{1}, \cdots, \chi_{8}\right\}=\operatorname{Irr}\left(B_{0}\right)$.

Case 1. $k^{\prime}\left(B_{0}\right)=7$ : We may assume $\chi_{1}(1)=\cdots=\chi_{7}(1)=1$ and $\chi_{8}(1)>1$. By Clifford's theorem, Proposition 1.3 and $(*)$, we have $\left.\chi_{1}\right|_{H}=\cdots=\left.\chi_{7}\right|_{H}=\tilde{\chi}_{1}$. Thus, by Proposition 1.4, $\left(\left.\chi_{8}\right|_{H}, \tilde{\chi}_{j}\right) \neq 0$ for $j=2, \cdots, 16$. Then we have a contradiction from Clifford's theorem and $\left(^{*}\right)$ by considering the degrees of $\tilde{\chi}_{j}$.

Case 2. $k^{\prime}\left(B_{0}\right)=5$ : We may assume $\chi_{i}(1)=1$ for $i=1, \cdots, 5$ and $\chi_{j}(1)>1$ for $j=6,7,8$. As in Case 1 we know $\left.\chi_{1}\right|_{H}=\cdots=\left.\chi_{5}\right|_{H}=\tilde{\chi}_{1}$. Since $k\left(B_{0}\right) \neq k\left(b_{0}\right)$, $B_{0} \cong b_{0}$. So that we get from Proposition 1.6 that $G \neq V H$ where $V$ is a subgroup of $G$ with $C_{G}(P)=P \times V$. Since $k^{\prime}\left(B_{0}\right)=5,|G: H|=5$ by Proposition 1.5. So, by Clifford's theorem and Proposition 1.4,

$$
\begin{gathered}
\left.\chi_{6}\right|_{H}=\tilde{\chi}_{2}+\cdots+\tilde{\chi}_{6},\left.\quad \chi_{7}\right|_{H}=\tilde{\chi}_{7}+\cdots+\tilde{\chi}_{11}, \\
\left.\chi_{8}\right|_{H}=\tilde{\chi}_{12}+\cdots+\tilde{\chi}_{16}
\end{gathered}
$$

for suitable indexing of $\tilde{\chi}_{2}, \cdots, \tilde{\chi}_{16}$. Hence we have a contradiction from Clifford's theorem and $\left(^{*}\right)$ by considering the degrees of $\tilde{\chi}_{j}$

Case 3. $k^{\prime}\left(B_{0}\right)=3$ : Let $\chi_{i}(1)=1$ for $i=1,2,3$ and $\chi_{j}(1)>1$ for $j=4, \cdots, 8$. As in Case 2, $|G: H|=3$. Then, by Proposition 1.4, for suitable indexing of $\tilde{\chi}_{2}, \cdots, \tilde{\chi}_{16}$, we get

$$
\begin{gathered}
\left.\chi_{4}\right|_{H}=\tilde{\chi}_{2}+\tilde{\chi}_{3}+\tilde{\chi}_{4},\left.\quad \chi_{5}\right|_{H}=\tilde{\chi}_{5}+\tilde{\chi}_{6}+\tilde{\chi}_{7},\left.\quad \chi_{6}\right|_{H}=\tilde{\chi}_{8}+\tilde{\chi}_{9}+\tilde{\chi}_{10} \\
\left.\chi_{7}\right|_{H}=\tilde{\chi}_{11}+\tilde{\chi}_{12}+\tilde{\chi}_{13},\left.\quad \chi_{8}\right|_{H}=\tilde{\chi}_{14}+\tilde{\chi}_{15}+\tilde{\chi}_{16}
\end{gathered}
$$

Then we have a contradiction as in Case 2.

Thus, $k\left(B_{0}\right)=16$. Let $\left\{\chi_{1}, \cdots, \chi_{16}\right\}=\operatorname{Irr}\left(B_{0}\right)$. Since $l\left(b_{z}\right)=1, \chi_{i}(z)= \pm 1$ for $i=$ $1, \cdots, 16$ from Lemma 1.9. Thus, we know from Clifford's theorem, Proposition 1.3 and $(*)$ that $\left.\chi_{i}\right|_{H} \in \operatorname{Irr}\left(b_{0}\right)$ for all $i=1, \cdots, 16$. Hence, by Proposition 1.4, we may assume that $\left.\chi_{i}\right|_{H}=\tilde{\chi}_{i}$ for all $i=1, \cdots, 16$. This shows $k^{\prime}\left(B_{0}\right)=1$. So that $B_{0} \cong b_{0}$ from Propositions 1.5 and 1.6. This completes the proof of the theorem.

Proposition 6.11. If $e=15$, then there is a basic set $W$ of $B_{0}$ such that $W$ contains the trivial Brauer character and the decomposition matrix of $B_{0}$ with respect to $W$ has the form

$$
\begin{aligned}
& 1_{G} \quad 1 \\
& \delta_{2} . \quad 0
\end{aligned}
$$

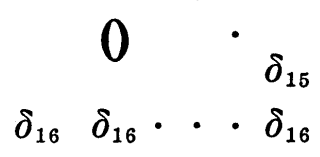


where $\delta_{i}= \pm 1$.

Proof. The proof is similar to that of Proposition 5.2 (cf. the case when $e=7$ in Proposition 5.2).

LEMMA 6.12. If $e=21$, then there is an involution $z \in P$ and there are two elements $s, t \in N_{G}(P)$ such that $N_{G}(P)=\left\langle s, t, C_{G}(P)\right\rangle, z^{s}=z$ and $z^{t}=z$.

Proof. Firstly, we want to prove that

there is an involution $u \in P$ and there are two elements $s, t \in N_{G}(P)$ such that $N_{G}(P)=\left\langle s, t, C_{G}(P)\right\rangle, s$ and $t$ have orders 3 and 7 modulo $C_{G}(P)$ respectively, and $u^{s}=u$.

We may assume $O(G)=1$ by the proof of Lemma 1.1. Since $S=O^{\prime}(G)$ is normal in $G, e(S)=1,7$ or 21 . When $e(S)=7$ or 21 , we get $\left(^{*}\right)$ from Proposition 1.10 and Lemma 1.12. Assume $e(S)=1$. Then $P$ is normal in $G$ and $|G: P|=21$. We can write $G=\langle s, t, P\rangle$ for $s, t \in G$ such that $s$ and $t$ have orders 3 and 7 modulo $P$, respectively. Clearly, there is an involution $y \in P$ with $y^{t}=y$. Suppose $x^{s} \neq x$ for all involutions $x \in P$. Then, $e\left(C_{G}(y)\right)=7$. By Proposition 5.2 and [10, Lemma 66.1], the Cartan matrix of $b_{y}$ has 2 as an elementary divisor of multiplicity 6 . Thus, by [5, (7G)], [18, Proposition 1.2] and [10, Theorem 65.4], we get $l\left(B_{0}\right) \geqq 7$ since all involutions in $G$ are conjugate. On the other hand, $l\left(B_{0}\right)=5$ since $G / P$ is noncyclic of order 21 (cf. Lemma 1.13). This is a contradiction. Hence we obtain $(*)$.

Next, we prove the lemma. There is an involution $z \in P$ with $z^{t}=z$. By $(*)$, there are other two involutions $v, w \in P$ such that $v^{s}=v, w^{s}=w$ and $u, v, w$ are all distinct. It suffices to show $z \in\{u, v, w\}$. Suppose $z \notin\{u, v, w\}$. Since $z^{s} \neq z$, we know that $\{1, u, v, w, z, u z, v z, w z\}$ is the set of all representatives of $\langle s\rangle$ conjugate classes of $P$. Since $u \neq z$, we get $u^{t} \neq u$. Thus, $\{1, u, u z, z\}$ is the set of all representatives of $\langle t\rangle$-conjugate classes of $P$. Hence, by elementary calculation, we get that $v \in\left\{u z, u^{t} z, \cdots, u^{t 6} z\right\}$. Hence no two elements in $\{u, v, z\}$ are conjugate in $G$. These show that all $G$-conjugate classes of $P$ are $\{1\},\{z\}$ $\left\{u, u^{t}, \cdots, u^{t^{6}}\right\}$ and $\left\{u z, u^{t} z, \cdots, u^{t^{6}} z\right\}$. Thus, $z^{s}=z$. This is a contradiction. This completes the proof.

LEMMA 6.13. If $e=21$, then $k\left(B_{0}\right)=16$ and $l\left(B_{0}\right)=5$.

PRoof. Let $s, t$ and $z$ be the same as in Lemma 6.12. Hence $s$ and $t$ have orders 3 and 7 modulo $C_{G}(P)$, respectively. There is an involution $x \in P$ with $x^{s}=x$ and $x^{t} \neq x$. Thus, $\{1, x, x z, z\}$ is the set of all representatives of conjugate 
classes of $G$ of 2-elements by [10, Lemma 18.5]. We may assume $O(G)=1$ by Lemma 1.1. By $Z^{*}$-theorem [10, Theorem 67.1], $z \in Z(G)$. These imply from [10, Theorems 68.4 and 65.4$]$ that $k\left(B_{0}\right)=2 l\left(B_{0}\right)+l\left(b_{x}\right)+l\left(b_{x z}\right)$. Since $e\left(C_{G}(x)\right)=3, l\left(b_{x}\right)$ $=3$ by Theorem 2.4(1). Similarly, $l\left(b_{x z}\right)=3$. Since $z \in Z(G)$, as in the proof of Lemma 2.2 we get from Lemma 4.1 and Proposition 1.2 that $l\left(B_{0}\right)=5$, so that $k\left(B_{0}\right)=16$.

Lemma 6.14. Assume $e=21, O(G)=1, O^{\prime}(G)=Z_{2} \times S L(2,8)$ and $G$ has a normal subgroup $H$ of odd prime index with $e(H)=7$. Then for any involution $z$ in $S L(2,8)$, we have

$$
\begin{array}{cl}
\chi_{1}(1)=\cdots=\chi_{6}(1)=1, & \chi_{7}(1)=\cdots=\chi_{12}(1)=7, \\
\chi_{13}(1)=\chi_{14}(1)=21, & \chi_{15}(1)=\chi_{16}(1)=27, \\
\chi_{1}(z)=\cdots=\chi_{6}(z)=1, & \chi_{7}(z)=\cdots=\chi_{12}(z)=-1, \\
\chi_{13}(z)=\chi_{14}(z)=-3, & \chi_{15}(z)=\chi_{16}(z)=3 .
\end{array}
$$

where $\left\{\chi_{1}=1_{G}, \chi_{2}, \cdots, \chi_{16}\right\}=\operatorname{Irr}\left(B_{0}\right)$.

Proof. Let $b_{0}=B_{0}(H), S=O^{\prime}(G)=\langle w\rangle \times S L(2,8)$ and $P=\langle w, x, y, z\rangle$ where $\langle x, y, z\rangle$ is a Sylow 2-subgroup of $S L(2,8)$. As in the proof of Lemma 5.3, G/H $=\langle r H\rangle$ for some $r \in N_{G}(P), C_{G}(P)=C_{H}(P)$ and $|G: H|=3$. We can write $N_{S}(P)$ $=\left\langle t, C_{S}(P)\right\rangle$ for some $t \in N_{S}(P)$. Since $P \cap Z(S)=\langle w\rangle$, it follows from Lemma 6.12 and $Z^{*}$-theorem [10, Theorem 67.1] that $w \in Z(G)$. Then, by the proof of Lemma 6.13, we may assume that $z^{t} \neq z, z^{r}=z$ and $l\left(b_{z}\right)=3$. By the proof of Lemma 2.2, $l\left(B_{0}\left(C_{S}(z)\right)\right)=1$. So that $C_{S}(z)$ is 2-nilpotent from [10, Corollary 65.3]. Hence $C_{G}(z)$ is solvable. Since $e\left(C_{G}(z)\right)=3$, by Proposition $6.1, b_{z} \cong B_{0}\left(P \cdot Z_{3}\right)$ where $P \cdot Z_{3}$ is a semi-direct product of its normal subgroup $P$ by $Z_{3}$ and it is not the direct product $P \times Z_{3}$. Then, by Lemma 6.5, we know the generalized decomposition numbers of $B_{0}$ relative to $z$. This implies

$$
\chi_{i}(z)= \begin{cases} \pm 1 & \text { for } i=1, \cdots, 12 \\ \pm 3 & \text { for } i=13, \cdots, 16\end{cases}
$$

for suitable indexing of $\chi_{2}, \cdots, \chi_{16}$. By Lemma 2.3, $b_{0} \cong B_{0}(S)$. So, by [10, Theorem 38.2],

$$
\begin{gathered}
\tilde{\chi}_{1}(1)=\tilde{\chi}_{2}(1)=1, \quad \tilde{\chi}_{3}(1)=\cdots \tilde{\chi}_{10}(1)=7, \\
\tilde{\chi}_{11}(1)=\cdots=\tilde{\chi}_{16}(1)=9, \\
\tilde{\chi}_{1}(z)=\tilde{\chi}_{2}(z)=1, \quad \tilde{\chi}_{3}(z)=\cdots=\tilde{\chi}_{10}(z)=-1, \\
\tilde{\chi}_{11}(z)=\cdots=\tilde{\chi}_{16}(z)=1
\end{gathered}
$$


where $\left\{\tilde{\chi}_{1}=1_{H}, \tilde{\chi}_{2}, \cdots, \tilde{\chi}_{16}\right\}=\operatorname{Irr}\left(b_{0}\right)$. We can write $C_{G}(P)=P \times V$. By Theorem 2.4 and Lemma 6.13 , we get $l\left(B_{0}\right) \neq l\left(b_{0}\right)=l\left(B_{0}(S)\right)$. Hence $B_{0} ¥ b_{0}$, so that $V H \neq G$ by Proposition 1.6. Thus, $|G: V H|=|G: H|=3$. Hence, by Proposition $1.5, k^{\prime}\left(B_{0}\right)$ is divisible by 3 . Since $|G: H|=3$, it follows from Frobenius reciprocity, Proposition 1.3 and $\left(^{* *}\right)$ that $k^{\prime}\left(B_{0}\right) \leqq 6$. By observing the conjugate classes of $G$ of 2-elements, we know $P \cap G^{\prime} \neq P$ from [10, Theorem 18.4]. Hence $\left|G: V G^{\prime}\right|$ is divisible by 2 . Thus, $k^{\prime}\left(B_{0}\right)=\left|G: V G^{\prime}\right|=6$ from Proposition 1.5. Then, by $\left(^{*}\right)$ and $(* *)$, we may assume that

$$
\left.\chi_{1}\right|_{H}=\left.\chi_{2}\right|_{H}=\left.\chi_{3}\right|_{H}=\tilde{\chi}_{1},\left.\quad \chi_{4}\right|_{H}=\left.\chi_{5}\right|_{H}=\left.\chi_{6}\right|_{H}=\tilde{\chi}_{2} .
$$

Similarly, we may assume that

$$
\begin{aligned}
& \left.\chi_{13}\right|_{H}=\tilde{\chi}_{5}+\tilde{\chi}_{6}+\tilde{\chi}_{7},\left.\quad \chi_{14}\right|_{H}=\tilde{\chi}_{8}+\tilde{\chi}_{9}+\tilde{\chi}_{10}, \\
& \left.\chi_{15}\right|_{H}=\tilde{\chi}_{11}+\tilde{\chi}_{12}+\tilde{\chi}_{13},\left.\quad \chi_{16}\right|_{H}=\tilde{\chi}_{14}+\tilde{\chi}_{15}+\tilde{\chi}_{16} .
\end{aligned}
$$

Hence we may assume that

$$
\left.\chi_{7}\right|_{H}=\left.\chi_{8}\right|_{H}=\left.\chi_{9}\right|_{H}=\tilde{\chi}_{3},\left.\quad \chi_{10}\right|_{H}=\left.\chi_{11}\right|_{H}=\left.\chi_{12}\right|_{H}=\tilde{\chi}_{4} .
$$

Therefore the lemma is proved by $\left.{ }^{(* *}\right)$.

Now, we state the next theorem which is one of the main results of this section.

THEOREM 6.15. Let $\bar{G}=G / O(G)$ and $S=O^{\prime}(\bar{G})$. If $G$ is nonsolvable, $e=21$ and $e(S) \neq 21$, then we have the following.

(i) $S \cong Z_{2} \times S L(2,8)$.

(ii) For any subnormal subgroup $\bar{L}$ of $\bar{G}$ of odd index with $e(\bar{L})=21$, $B_{0} \cong B_{0}(\bar{L})$.

Proof. We can assume $O(G)=1$ by Lemma 1.1. Hence $S=O^{\prime}(G)$.

(i) By Lemma 1.13, $e(S)=7$. Hence, by Proposition 1.10 and Lemma 1.12, $S \cong Z_{2} \times S L(2,8)$.

(ii) Firstly, we want to show that

$$
\left\{\begin{array}{l}
\text { if } L \text { is a normal subgroup of } G \text { such that }|G: L| \text { is an odd prime and } \\
e(L)=21 \text {, and if } H \text { is a normal subgroup of } L \text { such that }|L: H| \text { is an } \\
\text { odd prime and } e(H)=7 \text {, then } B_{0} \cong B_{0}(L) .
\end{array}\right.
$$

Let $b_{0}=B_{0}(L)$. By Lemma 6.13, $k\left(B_{0}\right)=k\left(b_{0}\right)=16$ and $l\left(B_{0}\right)=l\left(b_{0}\right)=5$. Let $S=O^{\prime}(G)$ $=\langle w\rangle \times S L(2,8)$ and $P=\langle w, x, y, z\rangle$ where $\langle x, y, z\rangle$ is a Sylow 2-subgroup of $S L(2,8)$. As in the proof of Lemma 6.14, 


$$
\chi_{i}(z)= \begin{cases} \pm 1 & \text { for } i=1, \cdots, 12 \\ \pm 3 & \text { for } i=13, \cdots, 16\end{cases}
$$

where $\left\{\chi_{1}, \cdots, \chi_{16}\right\}=\operatorname{Irr}\left(B_{0}\right)$. Let $\left\{\tilde{\chi}_{1}, \cdots, \tilde{\chi}_{16}\right\}=\operatorname{Irr}\left(b_{0}\right)$. By Lemma 6.14, we may assume that

$(* * *)$

$$
\begin{gathered}
\tilde{\chi}_{1}(1)=\cdots=\tilde{\chi}_{6}(1)=1, \quad \tilde{\chi}_{7}(1)=\cdots=\tilde{\chi}_{12}(1)=7, \\
\tilde{\chi}_{13}(1)=\tilde{\chi}_{14}(1)=21, \quad \tilde{\chi}_{15}(1)=\tilde{\chi}_{16}(1)=27, \\
\tilde{\chi}_{1}(z)=\cdots=\tilde{\chi}_{6}(z)=1, \quad \tilde{\chi}_{7}(z)=\cdots=\tilde{\chi}_{12}(z)=-1, \\
\tilde{\chi}_{13}(z)=\tilde{\chi}_{14}(z)=-3, \quad \tilde{\chi}_{15}(z)=\tilde{\chi}_{18}(z)=3 .
\end{gathered}
$$

Thus, as in the proof of Theorem 5.4, by $(* *),(* * *)$, Clifford's theorem and Proposition 1.4, we may assume that $\left.\chi_{i}\right|_{L}=\tilde{\chi}_{i}$ for all $i=1, \cdots, 16$. Hence we get $B_{0} \cong b_{0}$ by Corollary 1.7 . This proves $(*)$. Since $G / S$ is solvable by $[12$, Theorem], we can verify (ii).

REMARK 2. There is a finite group $G$ with elementary abelian Sylow 2-subgroups of order 16 such that $e(G)=21$ and $O^{\prime}(G / O(G)) \cong Z_{2} \times S L(2,8)$. We know it as in Remark 1 of $\S 2$.

Proposition 6.16. If $e=21$, then there is a basic set $W$ of $B_{0}$ such that $W$ contains the trivial Brauer character and the decomposition matrix of $B_{0}$ with respect to $W$ has the form

$\begin{array}{clllll}1_{G} & 1 & 0 & 0 & 0 & 0 \\ & \delta_{2} & 0 & 0 & 0 & 0 \\ 0 & \delta_{3} & 0 & 0 & 0 \\ 0 & \delta_{4} & 0 & 0 & 0 \\ 0 & 0 & \delta_{5} & 0 & 0 \\ & 0 & 0 & \delta_{6} & 0 & 0 \\ 0 & 0 & 0 & \delta_{7} & 0 \\ 0 & 0 & 0 & \delta_{8} & 0 \\ 0 & 0 & 0 & 0 & \delta_{9} \\ 0 & 0 & 0 & 0 & \delta_{10} \\ \delta_{11} & 0 & 0 & \delta_{11} & \delta_{11} \\ \delta_{12} & 0 & 0 & \delta_{12} & \delta_{12} \\ 0 & \delta_{13} & 0 & \delta_{13} & \delta_{13} \\ 0 & \delta_{14} & 0 & \delta_{14} & \delta_{14} \\ 0 & 0 & \delta_{15} & \delta_{15} & \delta_{15} \\ 0 & 0 & \delta_{16} & \delta_{16} & \delta_{16}\end{array}$


where $\delta_{i}= \pm 1$.

Proof. We can verify the proposition as in Proposition 5.2.

\section{References}

[1] Alperin, J.L., Isomorphic blocks. J. Algebra 43 (1976), 694-698.

[2] Bender, H., On groups with abelian Sylow 2-subgroups. Math. Z. 117 (1970), 164-176.

[3] Brauer, R., Some applications of the theory of blocks of characters of finite groups I-IV. J. Algebra 1 (1964), 152-167; ibid. 1 (1964), 307-334; ibid. 3 (1966), 225-255; ibid. 17 (1971), 489-521.

[4] Brauer, R., On blocks and sections in finite groups I-II. Amer. J. Math. 89 (1967), 1115-1136; ibid. 90 (1968), 895-925.

[5] Brauer, R., Defect groups in the theory of representations of finite groups. Illinois J. Math. 13 (1969), 53-73.

[6] Brauer, R., On 2-blocks with dihedral defect groups. Symposia Mathematica 13 (1974), 367-393.

[7] Brauer, R. and Nesbitt, C., On the modular characters of groups. Ann. of Math. (2) 42 (1941), 556-590.

[8] Curtis, C.W. and Reiner, I., Representation theory of finite groups and associative algebras. Interscience, New York, 1962.

[9] Dade, E. C., Blocks with cyclic defect groups. Ann. of Math. (2) 84 (1966), 20-48.

[10] Dornhoff, L., Group representation theory (parts A and B). Dekker, New York, 1971-72.

[11] Feit, W., Representations of finite groups (mimeographed notes). Yale University, Connecticut, 1969.

[12] Feit, W. and Thompson, J.G., Solvability of groups of odd order. Pacific J. Math. 13 (1963), 775-1029.

[13] Fong, P., On decomposition numbers of $J_{1}$ and $R(q)$. Symposia Mathematica 13 (1974), 415-422.

[14] Gorenstein, D., Finite groups. Harper and Row, New York, 1968.

[15] Huppert, B., Endliche Gruppen I. Springer, Berlin, 1967.

[16] Janko, Z., A new finite simple group with abelian Sylow 2-subgroups and its characterization. J. Algebra 3 (1966), 147-186.

[17] Janko, Z. and Thompson, J.G., On a class of finite simple groups of Ree. J. Algebra 4 (1966), 274-292.

[18] Olsson, J.B., On 2-blocks with quaternion and quasidihedral defect groups. J. Algebra 36 (1975), 212-241.

[19] Reynolds, W.F., A block correspondence and isometries of group characters. Math. Z. 113 (1970), 1-16.

[20] Walter, J. H., The characterization of finite groups with abelian Sylow 2-subgroups. Ann. of Math. (2) 89 (1969), 405-514.

[21] Ward, H.N., On Ree's series of simple groups. Trans. Amer. Math. Soc. 121 (1966), 62-89. 